\title{
Verification of joint input-state estimation for force identification by means of in situ measurements on a footbridge
}

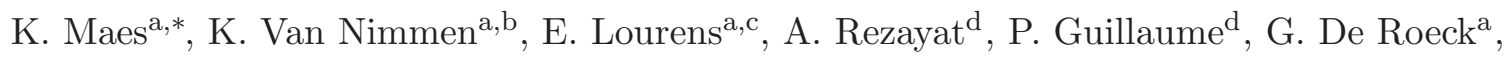 \\ G. Lombaert ${ }^{\mathrm{a}}$ \\ ${ }^{a} K U$ Leuven, Department of Civil Engineering, Leuven, Belgium \\ ${ }^{b}$ KU Leuven@KAHO Sint-Lieven, Department of Civil Engineering, Ghent, Belgium \\ ${ }^{c}$ Delft University of Technology, Faculty of Civil Engineering and Geosciences, Delft, The Netherlands \\ ${ }^{d}$ Vrije Universiteit Brussel, Department of Mechanical Engineering, Brussels, Belgium
}

\begin{abstract}
This paper presents a verification of a joint input-state estimation algorithm using data obtained from in situ experiments on a footbridge. The estimation of the input and the system states is performed in a minimum-variance unbiased way, based on a limited number of response measurements and a system model. A dynamic model of the footbridge is obtained using a detailed finite element model that is updated using a set of experimental modal characteristics. The joint inputstate estimation algorithm is used for the identification of two impact, harmonic, and swept sine forces applied to the bridge deck. In addition to these forces, unknown stochastic forces, such as wind loads, are acting on the structure. These forces, as well as measurement errors, give rise to uncertainty in the estimated forces and system states. Quantification of the uncertainty requires determination of the power spectral density of the unknown stochastic excitation, which is identified from the structural response under ambient loading. The verification involves comparing the estimated forces with the actual, measured forces. Although a good overall agreement is obtained between the estimated and measured forces, modeling errors prohibit a proper distinction between multiple forces applied to the structure for the case of harmonic and swept sine excitation.
\end{abstract}

Keywords: joint input-state estimation, force identification, data fusion, uncertainty quantification, application

\section{Introduction}

Knowledge of the loads applied to structures and the corresponding system response is very important for many engineering applications. However, in various cases, the dynamic forces acting on a structure cannot be obtained by direct measurements. For example, this is the case for wind

\footnotetext{
"Postprint submitted to Mechanical Systems and Signal Processing

wh Published version: K. Maes, K. Van Nimmen, E. Lourens, A. Rezayat, P. Guillaume, G. De Roeck, and G. Lombaert. Verification of joint input-state estimation for force identification by means of in situ measurements on a footbridge. Mechanical Systems and Signal Processing, 75:245-260, 2016.

http://dx.doi.org/10.1016/j.ymssp.2015.12.017

*Corresponding author. Tel.: +32 (0) 16322573.

Email address: kristof .maes@bwk.kuleuven.be (K. Maes)
} 
loads acting on a wind turbine. In addition, practical and economic considerations can prevent the response of a structure being measured at all physical locations. System inversion techniques address these problems by combining available vibration data from a limited number of sensors with the information obtained from a dynamic model of the structure, hereby estimating the forces acting on the structure and the response at unmeasured locations.

Many force identification algorithms have been proposed in the literature $[1,2,3,4,5,6$, 7]. These methods can be distinguished by the way they tackle the ill-posedness of the force identification problem [8]. Additionally, several state estimation algorithms have been proposed for linear as well as non-linear systems $[9,10]$. A common approach in state estimation consists of modeling the system input as zero mean Gaussian white noise and applying a Bayesian framework for state estimation $[11,12]$. In cases where state estimation is performed for uncertain dynamic systems, the system states are estimated together with the unknown system parameters, which is referred to in the literature as joint state and parameter estimation. Well known algorithms used for joint state and parameter estimation are the unscented Kalman filter (UKF) [13, 14], the extended Kalman filter (EKF) [15], and the particle filter $[14,16]$. A comprehensive overview of the current state of the art can be found in [17].

In order to overcome the assumption of white noise system input, which is often violated in practical applications, filtering methods in the presence of unknown inputs have been developed. The algorithms are often referred to as joint input-state estimation algorithms and combine both input and state estimation, e.g. [18, 19, 20, 21, 22, 23]. Recursive combined deterministic-stochastic approaches allow online joint input-state estimation, thereby accounting for measurement errors, modelling errors, and additional unknown vibration sources. Gillijns and De Moor [24] have proposed an algorithm where the input estimation is performed prior to the state estimation step. This algorithm was introduced to the field of structural dynamics by Lourens et al. [25], extending the use of the algorithm to reduced-order models. The algorithm is further extended in [26], for cases where accelerations are measured in the presence of unknown stochastic excitation. A similar approach was proposed by Niu et al. [27]. Alternatively, a classical Kalman filter can be used to jointly estimate the dynamic forces and system states, thus including the unknown forces in an augmented state vector [28]. It is noted that the aforementioned filtering approaches for joint input-state estimation require displacement or strain measurements in addition to acceleration measurements, in order to avoid erroneous low frequency components on the estimated input and states [29]. Following Chatzi and Fuggini [30], Naets et al. [31] propose including artificial white noise displacement measurements as observations, in addition to acceleration measurements, to avoid low frequency drift of the estimated input.

Verification of the force identification techniques proposed in the literature is, to date, mostly based on numerical simulations, where (idealized) measurement errors are incorporated by adding white noise to the simulated response signals, or to laboratory experiments. This paper presents a full-scale verification of the joint input-state estimation algorithm proposed in [26] using data obtained from an in situ experiment on a footbridge. The algorithm is used to identify the impact, harmonic, and swept sine excitations applied to the bridge deck. The estimated forces are verified by comparing them to the actual, measured forces.

The outline of the paper is as follows: Section 2 gives the extension of the joint input-state estimation algorithm; Section 3 discusses the setup of the experiments on the footbridge; Section 4 explains how a finite element model has been set up and updated, and shows the derivation of a state-space model representing the dynamic behavior of the structure; Section 5 presents a 
selection of data that allows for force identification; Section 6 quantifies the uncertainty introduced by measurement errors and unknown stochastic excitation of the structure, in addition to the forces that are to be identified; Section 7 discusses the results of the force identification; and Section 8 presents conclusions.

\section{Mathematical formulation}

Section 2.1 first gives a brief summary of the joint input-state estimation algorithm introduced in [26]. Next, Section 2.2 describes the approach for quantification of the estimation uncertainty originating from measurement errors and unknown stochastic excitation presented in [26]. This approach is applied in this paper to quantify the uncertainty in the estimated forces that results from measurement noise and wind loads acting on the footbridge.

\subsection{Joint input-state estimation algorithm}

Assume the following discrete-time combined deterministic-stochastic state-space description of a system:

$$
\begin{aligned}
\mathbf{x}_{[k+1]} & =\mathbf{A x}_{[k]}+\mathbf{B} \mathbf{p}_{[k]}+\mathbf{w}_{[k]} \\
\mathbf{d}_{[k]} & =\mathbf{G x}_{[k]}+\mathbf{J} \mathbf{p}_{[k]}+\mathbf{v}_{[k]}
\end{aligned}
$$

where $\mathbf{x}_{[k]} \in \mathbb{R}^{n_{\mathrm{s}}}$ is the state vector, $\mathbf{d}_{[k]} \in \mathbb{R}^{n_{\mathrm{d}}}$ is the output vector, assumed to be measured, and $\mathbf{p}_{[k]} \in \mathbb{R}^{n_{\mathrm{p}}}$ is the unknown input vector, with $n_{\mathrm{s}}$ the number of system states, $n_{\mathrm{d}}$ the number of outputs, and $n_{\mathrm{p}}$ the number of inputs. The system matrices $\mathbf{A}, \mathbf{B}, \mathbf{G}$, and $\mathbf{J}$ are assumed known. The expressions for the system matrices for modally reduced order models and full order models, e.g. based on the mass, damping, and stiffness matrices obtained from a finite element model, can be found in [25]. As an alternative to models based on first principles, models can be directly identified from experimental vibration data using system identification techniques, see for example [32, 33]. Throughout the derivation of the algorithm, it is assumed that the sensor network meets the conditions for instantaneous system inversion derived in [29].

The process noise vector $\mathbf{w}_{[k]} \in \mathbb{R}^{n_{\mathrm{s}}}$ and measurement noise vector $\mathbf{v}_{[k]} \in \mathbb{R}^{n_{\mathrm{d}}}$ both account for unknown excitation sources and modeling errors. The measurement noise vector $\mathbf{v}_{[k]}$ also accounts for measurement errors. The noise processes $\mathbf{w}_{[k]}$ and $\mathbf{v}_{[k]}$ are assumed to be zero mean and white, with known covariance matrices $\mathbf{Q}, \mathbf{R}$, and $\mathbf{S}$, defined by:

$$
\mathbb{E}\left[\left(\begin{array}{c}
\mathbf{w}_{[k]} \\
\mathbf{v}_{[k]}
\end{array}\right)\left(\begin{array}{ll}
\mathbf{w}_{[l]}^{\mathrm{T}} & \mathbf{v}_{[l]}^{\mathrm{T}}
\end{array}\right)\right]=\left[\begin{array}{cc}
\mathbf{Q} & \mathbf{S} \\
\mathbf{S}^{\mathrm{T}} & \mathbf{R}
\end{array}\right] \delta_{[k-l]}
$$

with $\mathbf{R}>0,\left[\begin{array}{cc}\mathbf{Q} & \mathbf{S} \\ \mathbf{S}^{\mathrm{T}} & \mathbf{R}\end{array}\right] \geq 0$, and $\delta_{[k]}=1$ for $k=0$ and 0 otherwise.

Joint input-state estimation consists of estimating the forces $\mathbf{p}_{[k]}$ and system states $\mathbf{x}_{[k]}$ from a set of response measurements $\mathbf{d}_{[k]}$. A state estimate $\hat{\mathbf{x}}_{[k \mid l]}$ is defined as an estimate of $\mathbf{x}_{[k]}$, given the output sequence $\mathbf{d}_{[n]}$, with $n=0,1, \ldots, l$. The corresponding error covariance matrix, denoted by $\mathbf{P}_{\mathrm{x}[k \mid l]}$, is defined as:

$$
\mathbf{P}_{\mathbf{x}[k \mid l]} \equiv \mathbb{E}\left\{\left(\mathbf{x}_{[k]}-\hat{\mathbf{x}}_{[k \mid l]}\right)\left(\mathbf{x}_{[k]}^{\mathrm{T}}-\hat{\mathbf{x}}_{[k \mid l]}^{\mathrm{T}}\right)\right\}
$$


where $\mathbb{E}\{\cdot\}$ indicates the expectation operator. An input estimate $\hat{\mathbf{p}}_{[k \mid l]}$ and its error covariance matrix $\mathbf{P}_{\mathrm{p}[k \mid l]}$ are defined similarly. The cross covariance matrices $\mathbf{P}_{\mathrm{xp}[k \mid l]}$ and $\mathbf{P}_{\mathrm{px}[k \mid l]}$ are defined as:

$$
\mathbf{P}_{\mathrm{xp}[k \mid l]}=\mathbf{P}_{\mathrm{px}[k \mid l]}^{\mathrm{T}} \equiv \mathbb{E}\left\{\left(\mathbf{x}_{[k]}-\hat{\mathbf{x}}_{[k \mid l]}\right)\left(\mathbf{p}_{[k]}^{\mathrm{T}}-\hat{\mathbf{p}}_{[k \mid l]}^{\mathrm{T}}\right)\right\}
$$

The filtering algorithm is initialized using an initial state estimate vector $\hat{\mathbf{x}}_{[0 \mid-1]}$ and its error covariance matrix $\mathbf{P}_{\mathbf{x}[0 \mid-1]}$. The estimate $\hat{\mathbf{x}}_{[0 \mid-1]}$ is assumed unbiased and independent of the noise processes $\mathbf{w}_{[k]}$ and $\mathbf{v}_{[k]}$ for all time steps $k$. The algorithm proceeds by computing the force and state estimates recursively in three steps: the input estimation step; the measurement update; and the time update. This is shown below.

Input estimation

$$
\begin{aligned}
\tilde{\mathbf{R}}_{[k]} & =\mathbf{G P}_{\mathrm{x}[k \mid k-1]} \mathbf{G}^{\mathrm{T}}+\mathbf{R} \\
\mathbf{M}_{[k]} & =\left(\mathbf{J}^{\mathrm{T}} \tilde{\mathbf{R}}_{[k]}^{-1} \mathbf{J}\right)^{-1} \mathbf{J}^{\mathrm{T}} \tilde{\mathbf{R}}_{[k]}^{-1} \\
\hat{\mathbf{p}}_{[k \mid k]} & =\mathbf{M}_{[k]}\left(\mathbf{d}_{[k]}-\mathbf{G} \hat{\mathbf{x}}_{[k \mid k-1]}\right) \\
\mathbf{P}_{\mathrm{p}[k \mid k]} & =\left(\mathbf{J}^{\mathrm{T}} \tilde{\mathbf{R}}_{[k]}^{-1} \mathbf{J}\right)^{-1}
\end{aligned}
$$

Measurement update

$$
\begin{aligned}
\mathbf{K}_{[k]} & =\mathbf{P}_{\mathbf{x}[k \mid k-1]} \mathbf{G}^{\mathrm{T}} \tilde{\mathbf{R}}_{[k]}^{-1} \\
\hat{\mathbf{x}}_{[k \mid k]} & =\hat{\mathbf{x}}_{[k \mid k-1]}+\mathbf{K}_{[k]}\left(\mathbf{d}_{[k]}-\mathbf{G} \hat{\mathbf{x}}_{[k \mid k-1]}-\mathbf{J} \hat{\mathbf{p}}_{[k \mid k]}\right) \\
\mathbf{P}_{\mathbf{x}[k \mid k]} & =\mathbf{P}_{\mathbf{x}[k \mid k-1]}-\mathbf{K}_{[k]}\left(\tilde{\mathbf{R}}_{[k]}-\mathbf{J} \mathbf{P}_{\mathrm{p}[k \mid k]} \mathbf{J}^{\mathrm{T}}\right) \mathbf{K}_{[k]}^{\mathrm{T}} \\
\mathbf{P}_{\mathrm{xp}[k \mid k]} & =\mathbf{P}_{\mathrm{px}[k \mid k]}^{\mathrm{T}}=-\mathbf{K}_{[k]} \mathbf{J} \mathbf{P}_{\mathrm{p}[k \mid k]}
\end{aligned}
$$

Time update

$$
\begin{aligned}
\hat{\mathbf{x}}_{[k+1 \mid k]} & =\mathbf{A} \hat{\mathbf{x}}_{[k \mid k]}+\mathbf{B} \hat{\mathbf{p}}_{[k \mid k]} \\
\mathbf{N}_{[k]} & =\mathbf{A} \mathbf{K}_{[k]}\left(\mathbf{I}_{n_{\mathrm{d}}}-\mathbf{J} \mathbf{M}_{[k]}\right)+\mathbf{B} \mathbf{M}_{[k]} \\
\mathbf{P}_{\mathbf{x}[k+1 \mid k]} & =\left[\begin{array}{ll}
\mathbf{A} & \mathbf{B}
\end{array}\right]\left[\begin{array}{ll}
\mathbf{P}_{\mathbf{x}[k \mid k]} & \mathbf{P}_{\mathbf{x p}[k \mid k]} \\
\mathbf{P}_{\mathbf{p x}[k \mid k]} & \mathbf{P}_{\mathrm{p}[k \mid k]}
\end{array}\right]\left[\begin{array}{l}
\mathbf{A}^{\mathrm{T}} \\
\mathbf{B}^{\mathrm{T}}
\end{array}\right]+\mathbf{Q}-\mathbf{N}_{[k]} \mathbf{S}^{\mathrm{T}}-\mathbf{S N}_{[k]}^{\mathrm{T}}
\end{aligned}
$$

The gain matrices $\mathbf{M}_{[k]}$ and $\mathbf{K}_{[k]}$ are determined such that the input estimates $\hat{\mathbf{p}}_{[k \mid k]}$ and state estimates $\hat{\mathbf{x}}_{[k \mid k]}$ are minimum variance and unbiased $[24,26]$. In the equations above, the system is assumed to be time-invariant. The algorithm can, however, be readily extended to time-variant systems by replacing the system matrices $\mathbf{A}, \mathbf{B}, \mathbf{G}$, and $\mathbf{J}$, with the system matrices $\mathbf{A}_{[k]}, \mathbf{B}_{[k]}$, $\mathbf{G}_{[k]}$, and $\mathbf{J}_{[k]}$, that depend on the time step $k$.

\subsection{Quantification of estimation uncertainty}

Estimation errors are due to the process noise $\mathbf{w}_{[k]}$ and measurement noise $\mathbf{v}_{[k]}$, which account for measurement errors, modeling errors, and unknown stochastic excitation. The noise processes $\mathbf{w}_{[k]}$ and $\mathbf{v}_{[k]}$ assumed for joint input-state estimation are zero mean, white, and stationary. The actual noise processes, however, do not generally meet the white noise assumption, such as in the case of wind loading. In this situation, the errors on the force and state estimates obtained from 
joint input-state estimation are no longer minimum variance and depend on the noise covariance matrices $\mathbf{Q}, \mathbf{R}$, and $\mathbf{S}$, that act as tuning parameters (see also [26]). In addition, the error covariance matrices $\mathbf{P}_{\mathrm{p}[k \mid k]}$ and $\mathbf{P}_{\mathrm{x}[k \mid k-m]}$ do not give the actual covariance of the error on the estimated quantities. This section briefly describes the method presented in [26] for quantification of the estimation uncertainty originating from measurement errors and unknown stochastic excitation. This approach is based on the steady-state transfer functions of the joint input-state estimation algorithm and the power spectral density functions of the measurement noise and the unknown stochastic forces, which are assumed stationary.

If the conditions for instantaneous system inversion presented in [29] are satisfied, the joint input-state estimation algorithm presented in Section 2.1 is stable and the gain matrices $\mathbf{M}_{[k]}$ and $\mathbf{K}_{[k]}$, as well as the error covariance matrices $\mathbf{P}_{\mathrm{p}[k \mid k]}, \mathbf{P}_{\mathrm{x}[k \mid k]}, \mathbf{P}_{\mathrm{x}[k \mid k-1]}, \mathbf{P}_{\mathrm{xp}[k \mid k]}$, and $\mathbf{P}_{\mathrm{px}[k \mid k]}$ evolve towards a steady-state value as the joint input-state estimation algorithm proceeds in time. The steady-state value of these matrices depends on the noise covariance matrices $\mathbf{Q}, \mathbf{R}$, and $\mathbf{S}$, and on the state-space system. When steady state is reached, the Fourier transform of the estimated

force vector $\hat{\mathbf{p}}_{[k \mid k]}$, denoted by $\hat{\mathbf{p}}(\omega)$, and the Fourier transform of the estimated state vectors $\hat{\mathbf{x}}_{[k \mid k]}$ and $\hat{\mathbf{x}}_{[k \mid k-1]}$, denoted by $\hat{\mathbf{x}}_{0}(\omega)$ and $\hat{\mathbf{x}}_{1}(\omega)$, respectively, are related to the Fourier transform of the output vector $\mathbf{d}_{[k]}$, denoted by $\mathbf{d}(\omega)$, through the following transfer functions:

$$
\left[\begin{array}{l}
\hat{\mathbf{p}}(\omega) \\
\hat{\mathbf{x}}_{0}(\omega) \\
\hat{\mathbf{x}}_{1}(\omega)
\end{array}\right]=\left[\begin{array}{l}
\mathbf{H}_{\hat{\mathrm{p}} \mathrm{d}}(\omega) \\
\mathbf{H}_{\hat{\mathrm{x}}_{0} \mathrm{~d}}(\omega) \\
\mathbf{H}_{\hat{\mathbf{x}}_{1} \mathrm{~d}}(\omega)
\end{array}\right] \mathbf{d}(\omega)
$$

where

$$
\left[\begin{array}{l}
\mathbf{H}_{\hat{\mathrm{p} d}}(\omega) \\
\mathbf{H}_{\hat{\mathrm{x}}_{0} \mathrm{~d}}(\omega) \\
\mathbf{H}_{\hat{\mathrm{x}}_{1} \mathrm{~d}}(\omega)
\end{array}\right]=\left[\begin{array}{lll}
\mathbf{I}_{n_{\mathrm{p}}} & \mathbf{0} & \mathbf{M}^{\mathrm{ss}} \mathbf{G} \\
\mathbf{K}^{\mathrm{ss}} \mathbf{J} & \mathbf{I}_{n_{\mathrm{s}}} & -\mathbf{I}_{n_{\mathrm{s}}}+\mathbf{K}^{\mathrm{ss}} \mathbf{G} \\
\mathbf{B} & \mathbf{A} & -\exp (i \omega \Delta t) \mathbf{I}_{n_{\mathrm{s}}}
\end{array}\right]^{-1}\left[\begin{array}{l}
\mathbf{M}^{\mathrm{ss}} \\
\mathbf{K}^{\mathrm{ss}} \\
\mathbf{0}
\end{array}\right]
$$

with $\mathbf{M}^{\mathrm{ss}}$ and $\mathbf{K}^{\mathrm{ss}}$ representing the steady-state gain matrices obtained from Eqs. (7) and (10), respectively (see also [34]). The sampling time step is $\Delta t$ and $\mathbf{I}_{n_{\mathrm{s}}} \in \mathbb{R}^{n_{\mathrm{s}} \times n_{\mathrm{s}}}$ and $\mathbf{I}_{n_{\mathrm{p}}} \in \mathbb{R}^{n_{\mathrm{p}} \times n_{\mathrm{p}}}$ are identity matrices.

When unknown stochastic forces are acting on the structure, in addition to the forces $\mathbf{p}_{[k]}$, and measurement errors are present, the process noise $\mathbf{w}_{[k]}$ and measurement noise $\mathbf{v}_{[k]}$ in Eqs. (1) and (2), respectively, are given by the following expressions:

$$
\begin{aligned}
\mathbf{w}_{[k]} & =\mathbf{B}^{\prime} \mathbf{p}_{\mathrm{S}[k]} \\
\mathbf{v}_{[k]} & =\mathbf{J}^{\prime} \mathbf{p}_{\mathrm{S}[k]}+\mathbf{v}_{\mathrm{M}[k]}
\end{aligned}
$$

where $\mathbf{p}_{\mathrm{S}[k]} \in \mathbb{R}^{n_{\mathrm{PS}}}$ is the vector representing the additional stochastic excitation, and the matrices $\mathbf{B}^{\prime} \in \mathbb{R}^{n_{\mathrm{s}} \times n_{\mathrm{PS}}}$ and $\mathbf{J}^{\prime} \in \mathbb{R}^{n_{\mathrm{d}} \times n_{\mathrm{P}_{\mathrm{S}}}}$ relate the state vector $\mathbf{x}_{[k+1]}$ and the output vector $\mathbf{d}_{[k]}$ to the vector of stochastic forces $\mathbf{p}_{\mathrm{S}[k]}$, respectively. The measurement noise due to measurement errors is $\mathbf{v}_{\mathrm{M}[k]}$. Under the assumption of stationary noise processes, the stochastic forces $\mathbf{p}_{\mathrm{S}[k]}$ are characterized by the autocorrelation (AC) function $\mathbf{R}_{\mathrm{p}_{\mathrm{S}} \mathrm{p}_{\mathrm{S}}}(\tau)$ and corresponding Power Spectral Density (PSD) function $\mathbf{S}_{\mathrm{p}_{\mathrm{S}} \mathrm{p}_{\mathrm{S}}}(\omega)$, that form the Wiener-Khinchin transformation pair:

$$
\begin{aligned}
& \mathbf{R}_{\mathrm{p}_{\mathrm{S}} \mathrm{p}_{\mathrm{S}}}(\tau) \equiv \mathbb{E}\left\{\mathbf{p}_{\mathrm{S}}(t+\tau) \mathbf{p}_{\mathrm{S}}^{\mathrm{T}}(t)\right\}=\frac{1}{2 \pi} \int_{-\infty}^{+\infty} \mathbf{S}_{\mathrm{P}_{\mathrm{S}} \mathrm{p}_{\mathrm{S}}}(\omega) \exp (i \omega \tau) d \omega \\
& \mathbf{S}_{\mathrm{P}_{\mathrm{S}} \mathrm{p}_{\mathrm{S}}}(\omega)=\int_{-\infty}^{+\infty} \mathbf{R}_{\mathrm{P}_{\mathrm{S}} \mathrm{p}_{\mathrm{S}}}(\tau) \exp (-i \omega \tau) d \tau
\end{aligned}
$$


where $i=\sqrt{-1}$. The discrete autocorrelation $\mathbf{R}_{\mathrm{p}_{\mathrm{S}} \mathrm{p}_{\mathrm{S}}[l]}$ is obtained as $\mathbf{R}_{\mathrm{p}_{\mathrm{S}} \mathrm{p}_{\mathrm{S}}}(l \Delta t)$, with $l$ the time lag. In case the stochastic forces can be modeled as finite bandwidth white noise, the autocorrelation $\mathbf{R}_{\mathrm{p}_{\mathrm{S}} \mathrm{p}_{\mathrm{S}}[l]}$ and PSD $\mathbf{S}_{\mathrm{p}_{\mathrm{S}} \mathrm{p}_{\mathrm{S}}}\left(\omega_{n}\right)$ are given by:

$$
\begin{aligned}
\mathbf{R}_{\mathrm{p}_{\mathrm{S}} \mathrm{p}_{\mathrm{S}}[l]} & =\mathbf{C}_{\mathrm{p}} \delta_{[l]} \\
\mathbf{S}_{\mathrm{P}_{\mathrm{S}} \mathrm{p}_{\mathrm{S}}}\left(\omega_{n}\right) & =\mathbf{C}_{\mathrm{p}} / F
\end{aligned}
$$

where $\mathbf{C}_{\mathrm{p}} \in \mathbb{R}^{n_{\mathrm{PS}} \times n_{\mathrm{PS}}}$ is the covariance matrix of the stochastic forces. For example, $\sigma_{\mathrm{p}_{\mathrm{S}}}^{2} \mathbf{I}_{n_{\mathrm{PS}}}$ represents the covariance matrix for $n_{\mathrm{pS}}$ independent stochastic forces with equal standard deviation $\sigma_{\mathrm{p}_{\mathrm{S}}}$. The sampling frequency used in the discretization process is $F, \omega_{n}=2 \pi(n-1) /(N \Delta t)$, and $\delta_{[l]}=1$ for $l=0$ and 0 otherwise.

The measurement errors $\mathbf{v}_{\mathrm{M}[k]}$ are defined similarly by the autocorrelation $\mathbf{R}_{\mathrm{v}_{\mathrm{M}} \mathrm{v}_{\mathrm{M}}[l]}$ and corresponding PSD $\mathbf{S}_{\mathrm{v}_{\mathrm{M}} \mathrm{v}_{\mathrm{M}}}\left(\omega_{n}\right)$, which, in case the measurement errors for all output signals can be modeled as finite bandwidth white noise, are given by:

$$
\begin{aligned}
\mathbf{R}_{\mathrm{v}_{\mathrm{M}} \mathrm{v}_{\mathrm{M}}[l]} & =\mathbf{R}_{\mathrm{M} \delta} \delta_{[l]} \\
\mathbf{S}_{\mathrm{v}_{\mathrm{M}} \mathrm{v}_{\mathrm{M}}}\left(\omega_{n}\right) & =\mathbf{R}_{\mathrm{M}} / F
\end{aligned}
$$

where $\mathbf{R}_{\mathrm{M}} \in \mathbb{R}^{n_{\mathrm{d}} \times n_{\mathrm{d}}}$ is the measurement error covariance matrix.

In absence of modeling errors, and assuming the stochastic forces $\mathbf{p}_{\mathrm{S}[k]}$ and the measurement errors $\mathbf{v}_{\mathrm{M}[k]}$ to be stationary and mutually uncorrelated (i.e. $\mathbb{E}\left\{\mathbf{p}_{\mathrm{S}[k]} \mathbf{v}_{\mathrm{M}[l]}^{\mathrm{T}}\right\}=\mathbf{0}$ ), the PSD function of the error on the estimated force vector $\tilde{\mathbf{p}}_{[k \mid k]}\left(\equiv \mathbf{p}_{[k]}-\hat{\mathbf{p}}_{[k \mid k]}\right)$ is found as [26]:

$$
\mathbf{S}_{\tilde{p} \tilde{p}}(\omega)=\mathbf{H}_{\hat{p} d}(\omega) \mathbf{H}_{\mathrm{dp}_{\mathrm{S}}}(\omega) \mathbf{S}_{\mathrm{p}_{\mathrm{S}} \mathrm{p}_{\mathrm{S}}}(\omega) \mathbf{H}_{\mathrm{dp}_{\mathrm{S}}}^{*}(\omega) \mathbf{H}_{\hat{\mathrm{p} d}}^{*}(\omega)+\mathbf{H}_{\hat{\mathrm{p}} \mathrm{d}}(\omega) \mathbf{S}_{\mathrm{v}_{\mathrm{M}} \mathrm{v}_{\mathrm{M}}}(\omega) \mathbf{H}_{\hat{\mathrm{p} d}}^{*}(\omega)
$$

where $\square^{*}$ denotes the Hermitian transpose of a matrix. The steady-state filter transfer function is $\mathbf{H}_{\hat{p} \mathrm{~d}}(\omega)$, defined in Eq. (18), and $\mathbf{H}_{\mathrm{dp}_{\mathrm{S}}}(\omega)$ is the transfer function matrix that relates the Fourier transform of the output vector $\mathbf{d}_{[k]}$ to the Fourier transform of the vector of stochastic forces $\mathbf{p}_{\mathrm{S}[k]}$ :

$$
\mathbf{H}_{\mathrm{dp}_{\mathrm{S}}}(\omega)=\mathbf{G}\left(\exp (i \omega \Delta t) \mathbf{I}_{n_{\mathrm{s}}}-\mathbf{A}\right)^{-1} \mathbf{B}^{\prime}+\mathbf{J}^{\prime}
$$

The autocorrelation function of the error is obtained as the inverse Fourier transform of the PSD function (Eq. (21)). The PSD function and autocorrelation function of the error on the estimated state vector $\tilde{\mathbf{x}}_{[k \mid k-m]}\left(\equiv \mathbf{x}_{[k]}-\hat{\mathbf{x}}_{[k \mid k-m]}\right)$ are similarly obtained. The PSD function of the error is given by the following expression:

$$
\begin{aligned}
\mathbf{S}_{\tilde{\mathrm{x}}_{m} \tilde{\mathrm{x}}_{m}}(\omega)= & \left(\mathbf{H}_{\mathrm{xp}_{\mathrm{S}}}(\omega)-\mathbf{H}_{\hat{\mathrm{x}}_{m} \mathrm{~d}}(\omega) \mathbf{H}_{\mathrm{dp}_{\mathrm{S}}}(\omega)\right) \mathbf{S}_{\mathrm{p}_{\mathrm{S}} \mathrm{p}_{\mathrm{S}}}(\omega)\left(\mathbf{H}_{\mathrm{xp}_{\mathrm{S}}}^{*}(\omega)-\mathbf{H}_{\mathrm{dp}_{\mathrm{S}}}^{*}(\omega) \mathbf{H}_{\hat{\mathrm{x}}_{m} \mathrm{~d}}^{*}(\omega)\right) \\
& +\mathbf{H}_{\hat{\mathrm{x}}_{m} \mathrm{~d}}(\omega) \mathbf{S}_{\mathrm{v}_{\mathrm{M}} \mathrm{v}_{\mathrm{M}}}(\omega) \mathbf{H}_{\hat{\mathrm{x}}_{m} \mathrm{~d}}^{*}(\omega)
\end{aligned}
$$

where $\mathbf{H}_{\hat{\mathrm{x}}_{m} \mathrm{~d}}(\omega)$ is the steady-state filter transfer function, defined in Eq. (18), and $\mathbf{H}_{\mathrm{xp}_{\mathrm{S}}}(\omega)$ is the transfer function matrix that relates the Fourier transform of the state vector $\mathbf{x}_{[k]}$, to the Fourier transform of the vector of stochastic forces $\mathbf{p}_{\mathrm{S}[k]}$ :

$$
\mathbf{H}_{\mathrm{xp}_{\mathrm{S}}}(\omega)=\left(\exp (i \omega \Delta t) \mathbf{I}_{n_{\mathrm{s}}}-\mathbf{A}\right)^{-1} \mathbf{B}^{\prime}
$$

Eqs. (27) and (29) yield the PSD function of the actual error on the estimated quantities for every possible choice of the noise covariance matrices $\mathbf{Q}, \mathbf{R}$, and $\mathbf{S}$. 


\section{Measurement setup}

The structure under consideration in this paper is a footbridge, located in Ninove (Belgium). The two-span cable-stayed steel bridge, shown in Fig. 1, has a main and secondary span of $36 \mathrm{~m}$ and $22.5 \mathrm{~m}$, respectively [35].

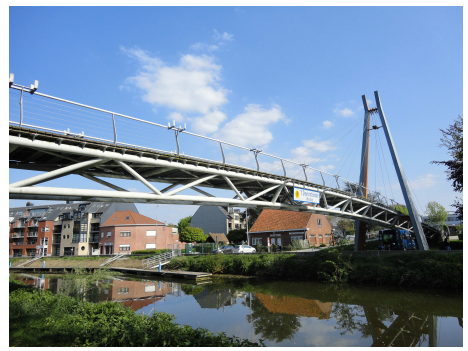

Fig. 1: The footbridge in Ninove, Belgium.

Three different types of excitation have been considered in the experiments: (1) ambient excitation, mainly consisting of wind loads, (2) excitation by hammer impacts, and (3) excitation by pneumatic actuators developed by the Acoustics and Vibration Research Group of the Vrije Universiteit Brussel [36]. During the experiments, the acceleration response of the footbridge has been recorded in three orthogonal directions at 12 locations on the bridge deck. In addition, the vertical displacement of the bridge deck has been recorded at two locations, as displacement data are needed to ensure the stability of the joint input-state estimation algorithm and the uniqueness of the force estimates obtained [29].

The vibration measurements have been performed using 12 wireless GeoSIG GMS-18 units and a National Instruments (NI) data acquisition system. The GMS-18 units include a high-sensitivity three-axial accelerometer for vibration monitoring. The NI system consists of a PXI-1050 chassis with four PXI-4472B modules and has been used to record data from four PCB 393B04 uniaxial accelerometers (sensitivity $\pm 1000 \mathrm{mV} / \mathrm{g}$ ), two PCB 086D50 instrumented hammers (mass $5.5 \mathrm{~kg}$ ), a PCB 222B load cell and a BD 5 load cell to measure the tension forces applied by the pneumatic actuators, and two AWLG 008M optical displacement sensors to measure the vertical displacements of the footbridge.

Fig. 2 gives an overview of the measurement locations. The GMS-18 units are positioned at the bridge deck surface. Each unit is tied to the bridge deck by a tension belt (Fig. 3a), thus avoiding loss of contact between the GMS-18 unit and the bridge deck. The uniaxial accelerometers at nodes 27 and 48 are mounted to a cross-beam at the lower side of the bridge deck using a magnet (Fig. 3b). The displacement of the footbridge is measured at nodes 27 and 40 in the vertical direction (i.e. the z-direction). The optical displacement sensors are mounted on top of a tripod support that is installed on firm ground underneath the bridge (Fig. 3c). Two uniaxial accelerometers are mounted orthogonally in the horizontal plane at the top of the tripod support at node 27 , in order to verify the assumption of a fixed point as reference for the displacement measurements. The hammer and muscle forces are applied at nodes 27 and 48 in the vertical direction.

A sampling frequency of $200 \mathrm{~Hz}$ and $1000 \mathrm{~Hz}$ is used for the GMS-18 units and the NI system, respectively. The GMS-18 units use an intelligent "Real Time Clock" (RTC) with a self-learning temperature compensation. For one of the units, hereafter referred to as the master unit, the RTC is synchronized with GPS. For the remaining units, the RTC is synchronized with the master unit 


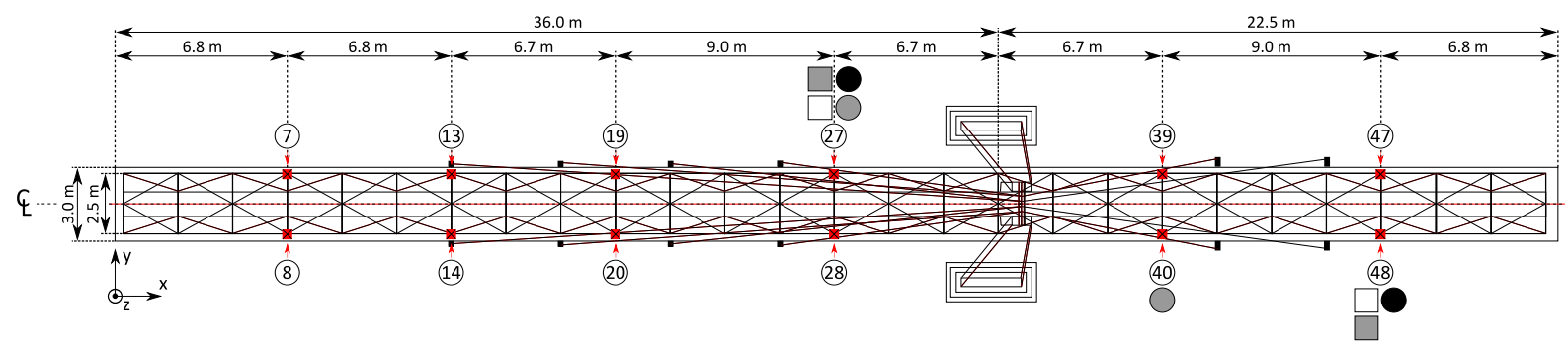

Fig. 2: Sensor configuration (white circle: GMS-18 unit, black circle: uniaxial accelerometer, gray circle: optical displacement sensor, white square: instrumented hammer, gray square: load cell).

(a)

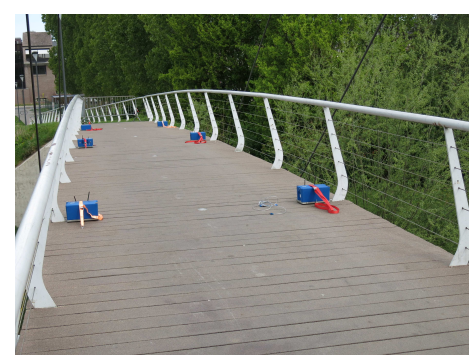

(b)

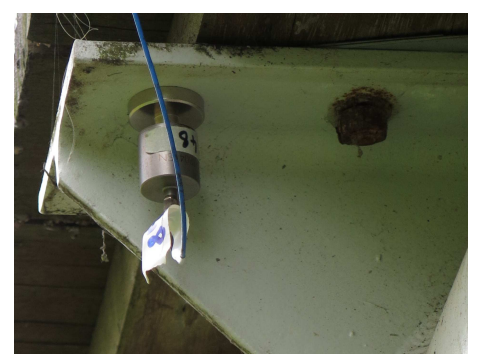

(c)

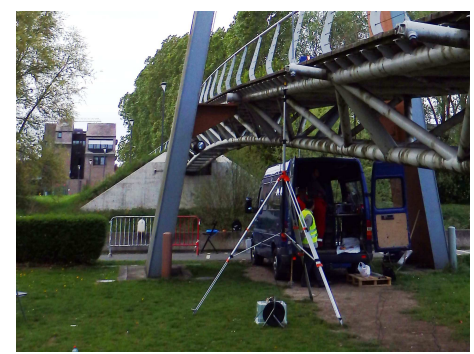

Fig. 3: Overview (a) GMS-units and connection to the bridge deck, (b) connection uniaxial accelerometer at node 48 to the cross-beam, and (c) tripod support for optical displacement sensor at node 40 .

on a wireless network connected through $433 \mathrm{MHz}$ time synchronisation modules. The GMS-18 acceleration data and the measurement data obtained from the NI system are synchronized by maximizing the correlation between the acceleration obtained from the GMS-18 unit at node 48 and the acceleration at node 48 obtained from the cabled uniaxial accelerometer.

The measured response and force signals used in the analyses are all digitally lowpass filtered by means of an eighth-order Chebyshev type I lowpass filter with a cut-off frequency of $16 \mathrm{~Hz}$, in both the forward and the reverse direction to remove all phase distortion, and then re-sampled at $40 \mathrm{~Hz}$. Next, the acceleration signals obtained from the NI system and the GMS-18 units are additionally digitally highpass filtered by means of a fifth-order Butterworth filter with cut-off frequencies of $0.5 \mathrm{~Hz}$ and $0.1 \mathrm{~Hz}$, respectively, in both the forward and the reverse direction. The aim of this filter is to remove the low frequency components from the signals that are contaminated by measurement noise. Finally, a detrend operation is applied to all acceleration signals to remove the (physically meaningless) DC component. The measured displacement signals for each experiment are relative to the displacement at the start of the experiment.

\section{System model}

The force identification is based on a state-space description of the system, given by Eqs. (1) and (2). The system model used in the present analysis is built from a detailed finite element model of the structure. Using a model based on first principles in the joint input state estimation procedure allows for reconstruction of the forces and the response at any location in the structure. In the case where models are directly identified from experimental vibration data [32, 33], the reconstruction of the forces and the response is restricted to those locations used in the experiments performed for the system identification. Models based on first principles are therefore more 
generally applicable, and can be used for a wide range of applications, such as the identification of distributed wind loads on wind turbines or moving traffic loads on bridges.

A modally reduced order model is used in this case, containing a limited number of structural modes. The composition of the system matrices $\mathbf{A}, \mathbf{B}, \mathbf{G}$, and $\mathbf{J}$ is outlined next.

\subsection{Finite element model}

A detailed finite element (FE) model of the footbridge has been developed using the FE program ANSYS [37]. The model is based on blueprints of the structure. The bridge deck is supported by a truss with a height of 1 meter (Fig. 1), composed of elements with a circular hollow section that are modeled using the ANSYS beam element BEAM4. The deck surface consists of wooden planks, modeled as added masses, and is supported by transverse beams that are welded to the truss. The latter are modeled using the BEAM4 element. The ANSYS shell element SHELL63 is used to model the pylons that have a rectangular section and a height of 16.5 meters. The pylon is assumed clamped at its support. The cables are modeled using the ANSYS 3D truss element LINK10, taking into account the effective stiffness of the cable based on the tensile force in the cable. At the abutments, the bridge deck is supported by neoprene bearings that are modeled as translational springs, using the ANSYS spring elements COMBIN14. The model has a total of 2421 nodes and 2508 elements. Fig. 4 shows a three-dimensional view of the ANSYS model.

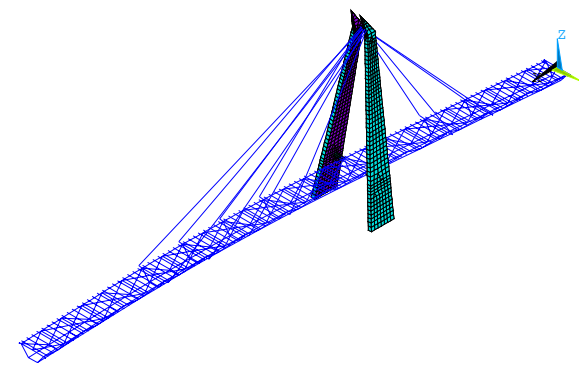

Fig. 4: 3D view of the ANSYS model.

The FE model of the footbridge is updated using a set of experimental modal parameters that have been obtained through a combination of output-only operational modal analysis (OMA) and input-output OMA with known exogenous inputs (OMAX), using hammer excitation and swept sine excitation by two pneumatic actuators. The response signals obtained from the OMA experiments are processed using the reference-based covariance-driven stochastic subspace identification (SSI-cov/ref) algorithm [38]. The response and force signals obtained from the OMAX experiments are processed using the reference-based data-driven combined deterministic-stochastic subspace identification (CSI-data/ref) algorithm [33]. In total, 18 modes of the bridge deck have been identified in the frequency range from $0 \mathrm{~Hz}$ to $20 \mathrm{~Hz}$. Table 1 gives the corresponding natural frequencies, the modal damping ratios, and a description of the mode shapes.

Comparison of the experimental modal parameters with the modal parameters obtained from the initial FE model shows some discrepancies. A model updating procedure is therefore performed. The updating parameters used in this analysis have been chosen based on a sensitivity analysis and include (1) the stiffnesses of the neoprene bearings, (2) the Young's modulus of the bridge deck, (3) the Young's modulus of the pylons, and (4) the effective Young's modulus of the cables. The natural frequencies and mode shapes corresponding to 14 identified modes are used as the 
observed quantities in the updating procedure, i.e. modes $1-5,7-9,11-13$, and $15-17$, listed in Table 1. The remaining modes, i.e. modes $6,10,14$, and 18, are used for cross validation of the model after updating. The updating is performed by minimizing the (weighted) least squares difference between the modal characteristics obtained from the FE model and the observed modal characteristics (natural frequencies and mode shapes). The optimization problem is solved using the non-linear least squares optimization routine (lsqnonlin) contained in MATLAB [39], where the FE analysis software ANSYS is applied (in batch mode) to compute the natural frequencies and mode shapes for a given set of parameter values.

Fig. 5 shows modes 1, 3, and 7 obtained from the updated FE model. Table 1 shows the modal characteristics obtained from the FE model after updating and comparison with the corresponding observed quantities. The relative error on the natural frequency $\varepsilon_{j}$ for mode $j$ is defined as $\varepsilon_{j}=\left(f_{j}-\tilde{f}_{j}\right) / \tilde{f}_{j}$, where $f_{j}$ is the undamped natural frequency corresponding to mode $j$, obtained from the $\mathrm{FE}$ model, and $f_{j}$ is the corresponding value obtained from the system identification. In general, very high MAC-values (MAC $\geq 0.89$ ) are obtained, both for the modes included in the model updating and the modes used for cross validation. This indicates a good overall agreement between the identified dynamic behavior of the footbridge and the behavior predicted by the model. Modeling errors cannot entirely be eliminated, however, as evidenced by the relatively large errors found for some of the natural frequencies obtained from the FE model.
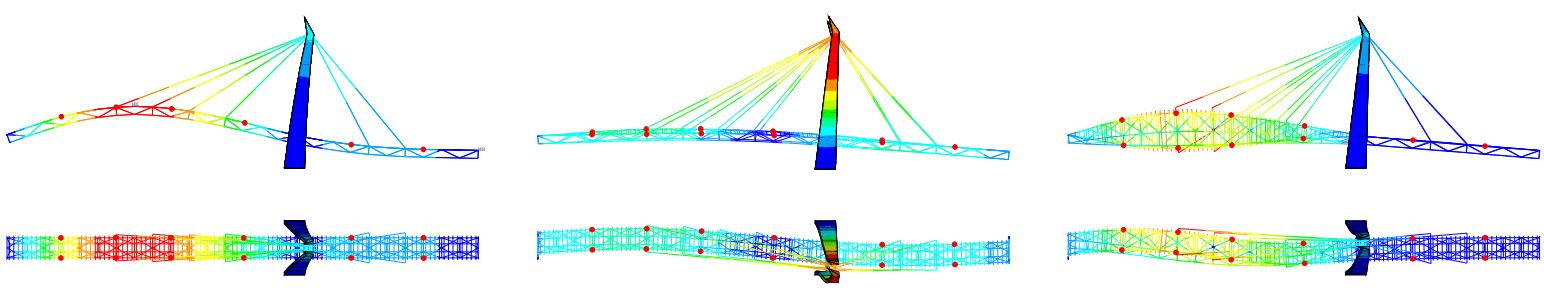

Fig. 5: Mode shape mode 1 (left), mode 3 (middle), and mode 7 (right) obtained from the updated FE model (top: side view, bottom: top view). The measurement locations are indicated by red dots.

\subsection{State-space description}

Next, a reduced-order discrete-time state-space model is constructed. When applying a zeroorder hold assumption on the input vector $\mathbf{p}_{[k]}$, the expressions for the state-feedback matrix $\mathbf{A}$ and the state-input matrix $\mathbf{B}$ in Eq. (1) are given by:

$$
\begin{aligned}
& \mathbf{A}=\exp \left(\left[\begin{array}{cc}
\mathbf{0} & \mathbf{I}_{n_{\mathrm{m}}} \\
-\boldsymbol{\Omega}^{2} & -\boldsymbol{\Gamma}
\end{array}\right] \Delta t\right) \\
& \mathbf{B}=\left(\mathbf{A}-\mathbf{I}_{n_{\mathrm{s}}}\right)\left[\begin{array}{cc}
\mathbf{0} & \mathbf{I}_{n_{\mathrm{m}}} \\
-\mathbf{\Omega}^{2} & -\boldsymbol{\Gamma}
\end{array}\right]^{-1}\left[\begin{array}{c}
\mathbf{0} \\
\boldsymbol{\Phi}^{\mathrm{T}} \mathbf{S}_{\mathrm{p}}
\end{array}\right]
\end{aligned}
$$

where $\mathbf{I}_{n_{\mathrm{m}}} \in \mathbb{R}^{n_{\mathrm{m}} \times n_{\mathrm{m}}}$ and $\mathbf{I}_{n_{\mathrm{s}}} \in \mathbb{R}^{n_{\mathrm{s}} \times n_{\mathrm{s}}}$ are identity matrices, with $n_{\mathrm{m}}$ the number of modes included in the system model and $n_{\mathrm{s}}$ the number of system states $\left(=2 n_{\mathrm{m}}\right) . \mathbf{S}_{\mathrm{p}} \in \mathbb{R}^{n_{\text {dof }} \times n_{\mathrm{p}}}$ is a selection matrix specifying the force locations, with $n_{\mathrm{p}}$ the number of forces and $n_{\text {dof }}$ the number of degrees of freedom in the FE model. $\boldsymbol{\Gamma} \in \mathbb{R}^{n_{\mathrm{m}} \times n_{\mathrm{m}}}$ is a diagonal matrix containing the terms $2 \xi_{j} \omega_{j}$ on its diagonal, where $\omega_{j}$ and $\xi_{j}$ are the natural frequency and modal damping ratio corresponding to 


\begin{tabular}{llllllll}
\hline$j$ & $\tilde{f}_{j}[\mathrm{~Hz}]$ & $\tilde{\xi}_{j}[\%]$ & $j_{\mathrm{fem}}$ & $f_{j}[\mathrm{~Hz}]$ & $\varepsilon_{j}[\%]$ & MAC [-] & Description \\
\hline 1 & 2.93 & 1.16 & 2 & 3.07 & 4.70 & 1.00 & 1st lateral bending main span \\
2 & 2.97 & 0.39 & 1 & 2.87 & -3.64 & 1.00 & 1st vertical bending main span \\
3 & 3.81 & 0.77 & 3 & 3.73 & -2.11 & 0.99 & 1st combined lateral bending \\
4 & 5.79 & 1.04 & 4 & 5.50 & -4.98 & 0.89 & 1st lateral bending secondary span \\
5 & 6.00 & 0.52 & 5 & 5.81 & -3.09 & 0.98 & 1st vertical bending secondary span \\
$6^{\dagger}$ & 7.06 & 0.20 & 7 & 7.07 & 0.08 & 0.94 & 1st torsional main span \\
7 & 7.27 & 1.26 & 6 & 6.84 & -5.95 & 0.96 & 2nd lateral bending main span \\
8 & 8.02 & 0.56 & 8 & 7.62 & -5.00 & 0.99 & 2nd vertical bending main span \\
9 & 9.83 & 0.73 & 11 & 9.97 & 1.38 & 0.94 & 2nd combined lateral bending \\
$10^{\dagger}$ & 11.06 & 1.28 & 12 & 10.80 & -2.39 & 0.96 & 1st torsional secondary span \\
11 & 11.44 & 2.09 & 13 & 11.60 & 1.38 & 0.94 & 2nd torsional main span \\
12 & 12.57 & 1.40 & 14 & 12.92 & 2.72 & 0.97 & 3rd combined lateral bending \\
13 & 13.59 & 0.41 & 15 & 13.07 & -3.85 & 0.98 & 3rd vertical bending main span \\
$14^{\dagger}$ & 14.08 & 0.47 & 16 & 14.07 & -0.12 & 0.93 & 3rd lateral bending main span \\
15 & 14.72 & 0.34 & 17 & 14.18 & -3.68 & 0.98 & 2nd vertical bending secondary span \\
16 & 16.20 & 0.94 & 19 & 16.84 & 3.98 & 0.97 & 4th lateral bending main span \\
17 & 17.55 & 1.33 & 21 & 18.71 & 6.61 & 0.90 & 2nd torsional secondary span \\
$18^{\dagger}$ & 18.63 & 0.68 & 20 & 17.86 & -4.17 & 0.93 & 4th vertical bending main span \\
\hline
\end{tabular}

Table 1: Comparison of the experimentally identified modal characteristics and the modal characteristics calculated from the updated FE model $\left(j\right.$ : No. identified mode, $\tilde{f}_{j}$ : identified undamped natural frequency, $\tilde{\xi}_{j}$ : identified modal damping ratio, $j_{\text {fem }}$ : No. corresponding mode updated FE model, $f_{j}$ : undamped natural frequency FE model, $\varepsilon_{j}$ : relative error $f_{j}$ w.r.t $\tilde{f}_{j}$, MAC: MAC-value). The identified modes indicated with a dagger were not included in the updating but were rather used for cross validation.

mode $j$, respectively. $\Omega \in \mathbb{R}^{n_{\mathrm{m}} \times n_{\mathrm{m}}}$ is a diagonal matrix as well, containing the natural frequencies $\omega_{j}$ on its diagonal, and $\boldsymbol{\Phi} \in \mathbb{R}^{n_{\mathrm{dof}} \times n_{\mathrm{m}}}$ is a matrix containing the mass normalized mode shapes $\phi_{j}$ as columns. The model includes all bending modes of the bridge deck with natural frequencies that fall within the frequency range $0 \mathrm{~Hz}$ to $20 \mathrm{~Hz}$, i.e. the 18 modes listed in Table $1\left(n_{\mathrm{m}}=18\right)$. For each mode, the mass normalized mode shape of the FE model is used. The natural frequency and modal damping ratio are taken as the experimentally identified values.

In general, the expressions for the state-output matrix $\mathbf{G}$ and the direct transmission matrix $\mathbf{J}$ in Eq. (2) do not depend on the time discretization scheme, and are given by:

$$
\begin{aligned}
\mathbf{G} & =\left[\begin{array}{ll}
\mathbf{S}_{\mathrm{d}, \mathrm{d}} \boldsymbol{\Phi}-\mathbf{S}_{\mathrm{d}, \mathrm{a}} \boldsymbol{\Phi} \boldsymbol{\Omega}^{2} & \mathbf{S}_{\mathrm{d}, \mathrm{v}} \boldsymbol{\Phi}-\mathbf{S}_{\mathrm{d}, \mathrm{a}} \boldsymbol{\Phi} \boldsymbol{\Gamma}
\end{array}\right] \\
\mathbf{J} & =\left[\begin{array}{ll}
\mathbf{S}_{\mathrm{d}, \mathrm{a}} \boldsymbol{\Phi} \boldsymbol{\Phi}^{\mathrm{T}} \mathbf{S}_{\mathrm{p}} &
\end{array}\right]
\end{aligned}
$$

where $\mathbf{S}_{\mathrm{d}, \mathrm{a}}, \mathbf{S}_{\mathrm{d}, \mathrm{v}}$, and $\mathbf{S}_{\mathrm{d}, \mathrm{d}} \in \mathbb{R}^{n_{\mathrm{d}} \times n_{\mathrm{dof}}}$ are selection matrices indicating the degrees of freedom corresponding to the acceleration, velocity, and displacement or strain measurements, respectively, with $n_{\mathrm{d}}$ the number of outputs. The output vector $\mathbf{d}_{[k]}$ is composed of $n_{\mathrm{d}, \mathrm{d}}$ displacement or strain measurements, $n_{\mathrm{d}, \mathrm{v}}$ velocity measurements, and $n_{\mathrm{d}, \mathrm{a}}$ acceleration measurements, where $n_{\mathrm{d}}$ represents the sum of $n_{\mathrm{d}, \mathrm{d}}, n_{\mathrm{d}, \mathrm{v}}$, and $n_{\mathrm{d}, \mathrm{a}}$.

\subsection{Forward calculations}

The quality of the system model presented in Section 4.2 is assessed by comparing the predicted response to the actual measured response of the footbridge obtained during one of the experiments. The measured hammer impacts at nodes 27 and 48, shown in Fig. 6, are applied as inputs to the 
system model described by Eqs. (1) and (2), disregarding the process noise $\mathbf{w}_{[k]}$ and measurement noise $\mathbf{v}_{[k]}$. Although these hammer impacts are considered as the only inputs in the calculations, the measured response also contains the influence of wind excitation. This should be kept in mind when comparing the measured and simulated response signals. The low frequency content from the measured displacement signals, which is mainly attributed to the wind loads, is removed by applying a fifth-order Butterworth highpass filter with a cut-off frequency of $0.2 \mathrm{~Hz}$ in both the forward and the reverse direction, hereafter performing a detrend operation.

Fig. 7 compares the measured and simulated vertical displacements at nodes 27 and 40, and the vertical and lateral accelerations at node 19. A window length of 1024 samples and an overlap of $66 \%$ are used for the calculation of the averaged amplitude of the frequency spectrum. Hammer impacts excite the entire frequency range of interest. After the impact, the response is dominated by free vibration at the natural frequencies of the structure. From the figures, very good overall agreement between the measured and simulated response signals is seen. The differences occurring between the measured and simulated response are mostly due to incomplete removal of the response due to ambient excitation, modeling errors, and, to a minor extent, sensor noise. The discrepancy between the measured and the simulated displacements observed in Figs. 7c and $7 \mathrm{f}$ at frequencies below the lowest natural frequency of the footbridge is most probably due to the quasi-static response to wind loads. Wind loads are also believed to be the cause of the discrepancy between the measured and simulated lateral acceleration at node 19 at frequencies below $4 \mathrm{~Hz}$ (Fig. 7l). The small peak in the frequency spectrum of the measured displacement at node 27 in Fig. $7 \mathrm{c}$, occurring at a frequency of about $2.65 \mathrm{~Hz}$, is due to spurious horizontal motion of the tripod supporting the displacement sensor. Since the displacements are measured relative to the position of the support, motion of the support is recorded, whereas a fixed support is assumed in the simulations. The same holds for the displacement sensor at node 40, where the horizontal motion of the short tripod gives rise to the peak in the frequency spectrum of the measured displacement (Fig. 7f), at a frequency of about $7 \mathrm{~Hz}$.
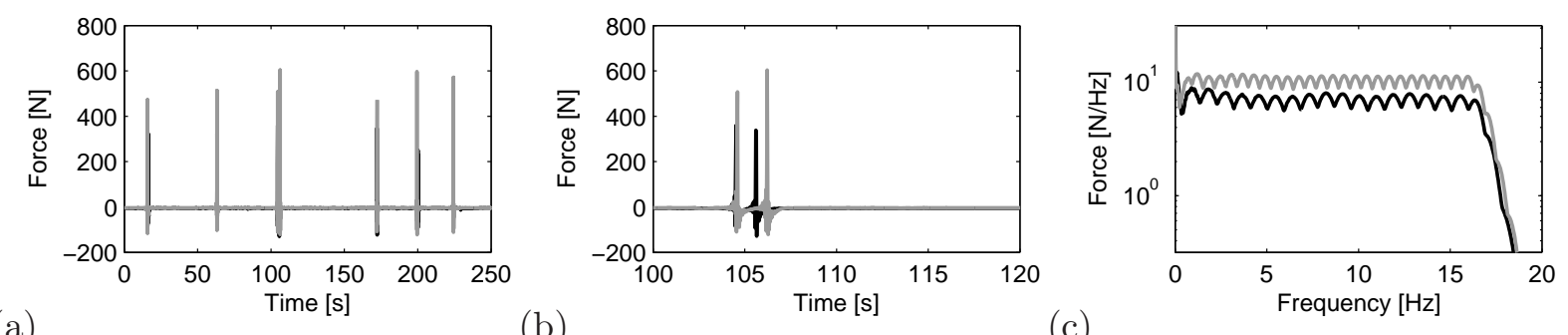

(a)

Fig. 6: (a) Time history, (b) detail of the time history, and (c) averaged amplitude of the narrow band frequency spectrum of the hammer forces applied at node 27 (black) and node 48 (gray).

\section{Selection of data for force identification}

Determination of the sensor configuration for force identification requires the meeting of the conditions for instantaneous system inversion [29]. The invertibility of a linear system model in general depends on three conditions. Firstly, the dynamic forces and/or corresponding states must be identifiable from the data. Secondly, the system inversion algorithm must be stable, such that small perturbations in the data do not give rise to unbounded errors on the identified forces and 


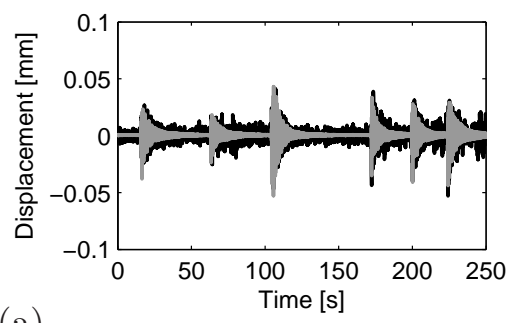

(a)

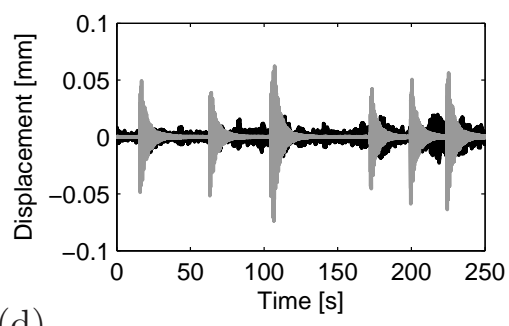

(d)

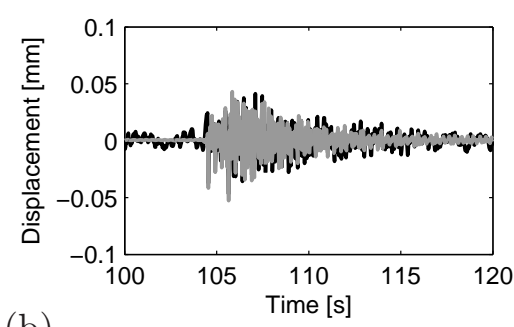

(b)

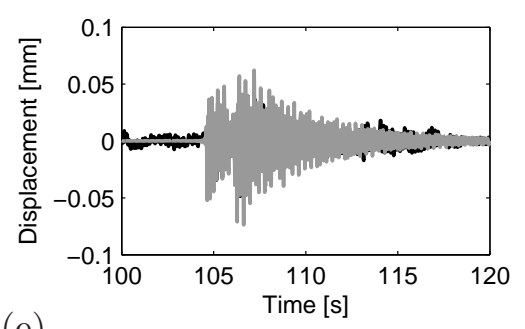

(e)

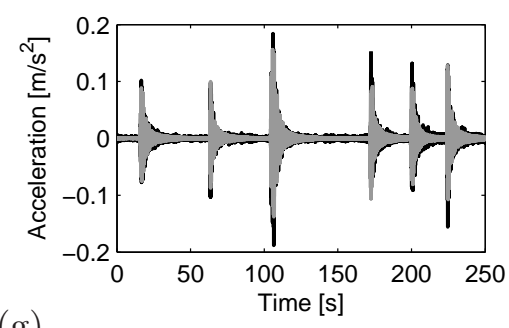

(g)

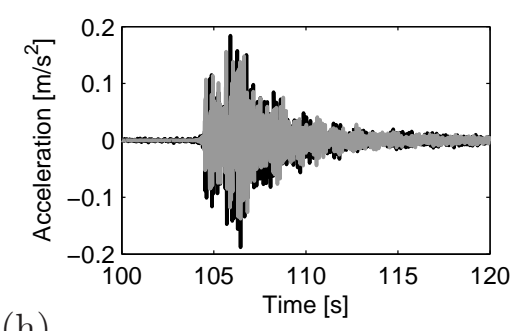

(h)

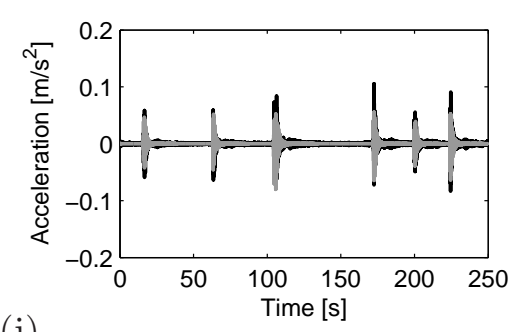

(j)

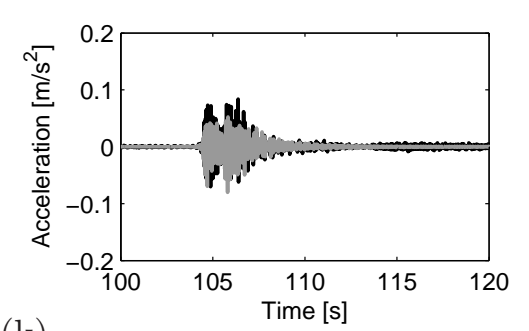

(k)

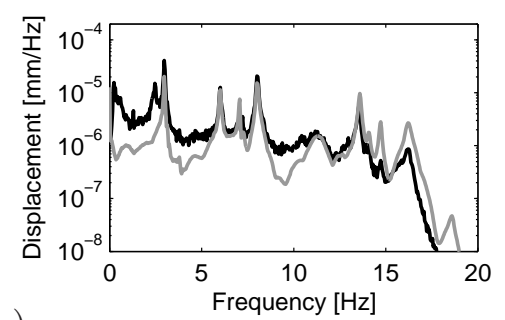

(c)

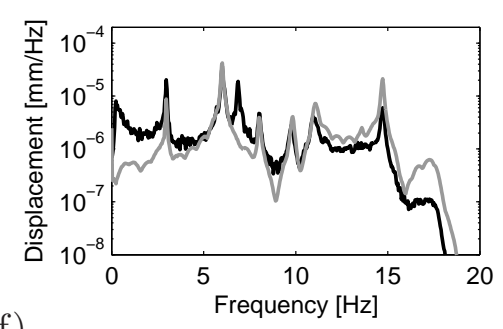

(f)

(i)
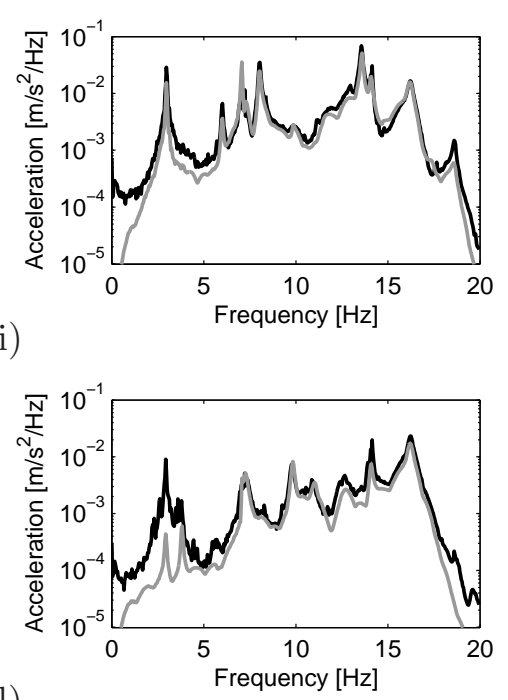

(1)

Fig. 7: Time history (left), detail of the time history (middle), and averaged amplitude of the narrow band frequency spectrum (right) of the vertical displacements at node $27((\mathrm{a})-(\mathrm{c}))$ and node $40((\mathrm{~d})-(\mathrm{e}))$, and the vertical ((g) (i)) and lateral $((\mathrm{j})-(\mathrm{l}))$ accelerations at node 19. The measured response is shown in black, the simulated response is shown in gray.

the system states. Thirdly, the estimates must be uniquely defined by the data. The general conditions for system inversion were recently translated into a number of requirements on the sensor network, i.e., sensor types, sensor locations, and number of sensors, for the specific case of linear modally reduced order models [29]. The invertibility conditions, derived assuming no noise, are necessary but not sufficient for guaranteeing that the forces and system states can be identified in the presence of noise.

In this study, the aim is to estimate vertical forces at nodes 27 and 48 (see Fig. 2), denoted by $\mathrm{p} 27 \mathrm{z}$ and $\mathrm{p} 48 \mathrm{z}$. A selection of data is made from the complete data set, including all response measurements on the bridge deck as listed in Section 3. The displacements are denoted by d27z and $d 40 z$, the accelerations obtained from the NI system by a27ni and a48ni. The accelerations 
obtained from the GMS-18 units are denoted by aj $\zeta$, where $j$ refers to the node number in the measurement grid, and $\zeta$ denotes the measurement direction (y or $\mathrm{z}$ ).

Using the techniques proposed in [29], a minimum subset of output data is determined, which allows for the estimation of the forces. Since force identification is aimed at, the system states as such are not of interest, and system observability, which requires that all system states are observed from the data, is not implemented. In addition, the force locations and directions are known. This means that system controllability, which requires that all system states can be controlled by the input, is not relevant. In this case, at least two $\left(n_{\mathrm{p}}\right)$ accelerations and two $\left(n_{\mathrm{p}}\right)$ displacements are required to ensure a coupling between the estimated forces and the measured acceleration and displacement data, respectively, through two $\left(n_{\mathrm{p}}\right)$ modes. The two displacements, $\mathrm{d} 27 \mathrm{z}$ and $\mathrm{d} 40 \mathrm{z}$, have to be included in the data vector in order to obtain a stable system inverse with a unique solution. Additional accelerations are required for instantaneous system inversion. In the following, the data used for joint input-state estimation consists of two collocated acceleration measurements a27ni and a48ni and two displacement measurements d27z and d40z. For this data set, all invertibility conditions are met, and these will still hold when more measurements are added. The reader is referred to [29] for detailed information on the design of the sensor network.

\section{Quantification of uncertainty in the estimated forces}

The forward calculations in Section 4.3 have shown good agreement between the true dynamic behavior of the structure and the behavior predicted by the system model. The differences between the measured and simulated response are mostly due to ambient excitation. In this case, the noise processes $\mathbf{w}_{[k]}$ and $\mathbf{v}_{[k]}$ in Eqs. (1) and (2) will, therefore, primarily cover ambient excitation. Since the ambient excitation includes wind loading, which does not generally correspond to white noise, the uncertainty in the estimated forces is calculated from Eq. (27) (see Section 2.2). This calculation requires the PSD $S_{\mathrm{P}_{\mathrm{S}} \mathrm{p}_{\mathrm{S}}}(\omega)$ of the ambient forces. The estimation of this PSD is described below.

The PSD of the ambient forces acting on the structure $\mathbf{S}_{\mathrm{p}_{\mathrm{S}} \mathrm{p}_{\mathrm{S}}}(\omega)$ is estimated from the measured response due to wind loadings. The response $\mathbf{d}_{[k]}$ assumed for estimating the PSD consists of the vertical and lateral accelerations obtained from the twelve GMS-18 units (Fig. 8) that have been measured for a period of 27 minutes. The cross-PSD of the output signals, $\mathbf{S}_{\mathrm{dd}}(\omega)$, is estimated using the average periodogram method [40], by subdividing the data series in blocks of 40 seconds. As an illustration, the auto-PSD and cross-PSD of the measured vertical and lateral acceleration at node 27 are shown in Fig. 9.

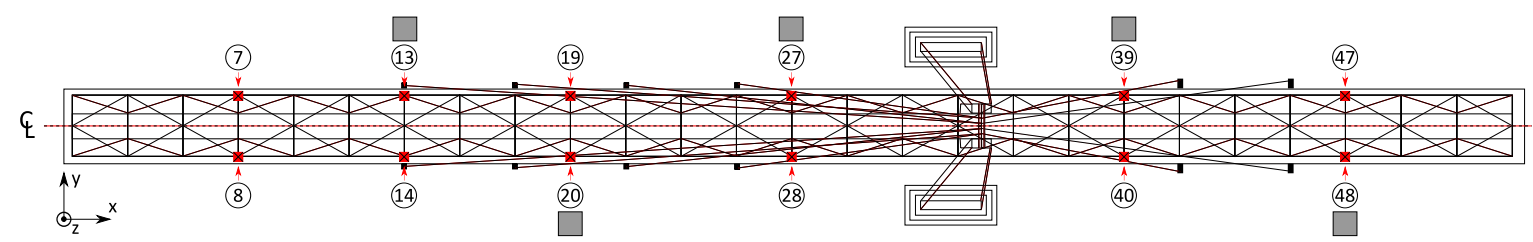

Fig. 8: Sensor configuration and force locations assumed for the uncertainty quantification procedure. A circle corresponds to a measured vertical $(\mathrm{z}$ ) and lateral (y) acceleration obtained from a GMS-18 unit. A gray square corresponds to a vertical (z) and a lateral (y) nodal (stochastic) force.

Ten stochastic forces are assumed in the form of concentrated point loads at nodes 13, 20, 27, 39, and 48, acting in the vertical and lateral directions (see Fig. 8). These forces are equivalent forces representing additional unknown excitations. The ten forces $\mathbf{p}_{\mathrm{S}[k]}$ are able to jointly excite all 


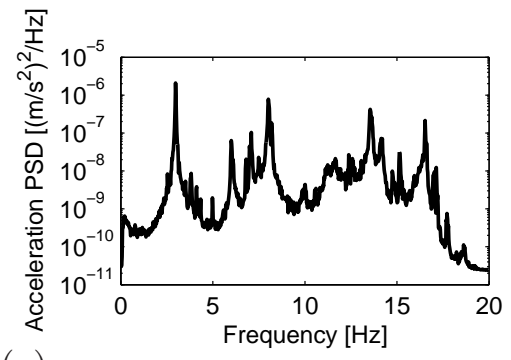

(a)

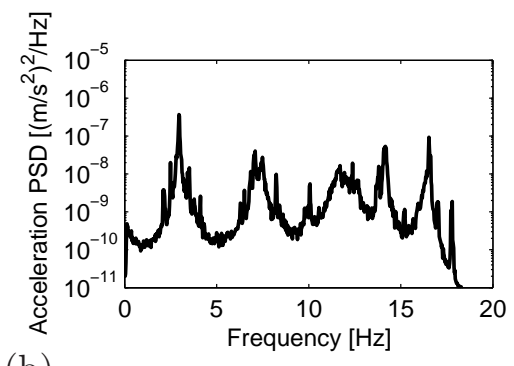

(b)

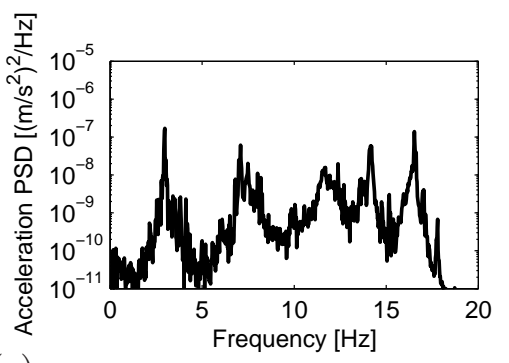

(c)

Fig. 9: Auto-PSD of (a) the vertical acceleration and (b) the lateral acceleration at node 27, and (c) amplitude of the cross-PSD of the vertical acceleration and the lateral acceleration at node 27.

modes included in the system model. Both vertical and lateral forces are assumed, since wind loads act in both the vertical and lateral directions on the bridge deck. The PSD of the stochastic forces, $\mathbf{S}_{\mathrm{p}_{\mathrm{S}} \mathrm{p}_{\mathrm{S}}}(\omega)$, is obtained from the cross-PSD of the output signals, $\mathbf{S}_{\mathrm{dd}}(\omega)$, using the Moore-Penrose pseudo-inverse (denoted by ${ }^{\dagger}$ ) of the FRF matrix $\mathbf{H}_{\mathrm{dp}_{\mathrm{S}}}(\omega)$ :

$$
\mathbf{S}_{\mathrm{p}_{\mathrm{S}} \mathrm{p}_{\mathrm{S}}}(\omega)=\mathbf{H}_{\mathrm{dp}_{\mathrm{S}}}^{\dagger}(\omega) \mathbf{S}_{\mathrm{dd}}(\omega) \mathbf{H}_{\mathrm{dp}_{\mathrm{S}}}^{* \dagger}(\omega)
$$

Fig. 10 shows the auto-PSD and cross-PSD of the estimated vertical and lateral forces at node 39. The equivalent stochastic forces are dominated by low frequency components. This is expected, since the ambient excitation primarily consists of wind loads. The estimated force PSD is not flat, confirming that the assumption of white noise is not valid. It is also observed from Fig. 10 that the auto-PSD of the lateral force at node 39 is generally more than one order of magnitude larger than the auto-PSD of the vertical force at node 39. This is expected, since the wind loads mainly act in a horizontal direction on the bridge deck (see also ENV 1991-2-4:1995 [41]).

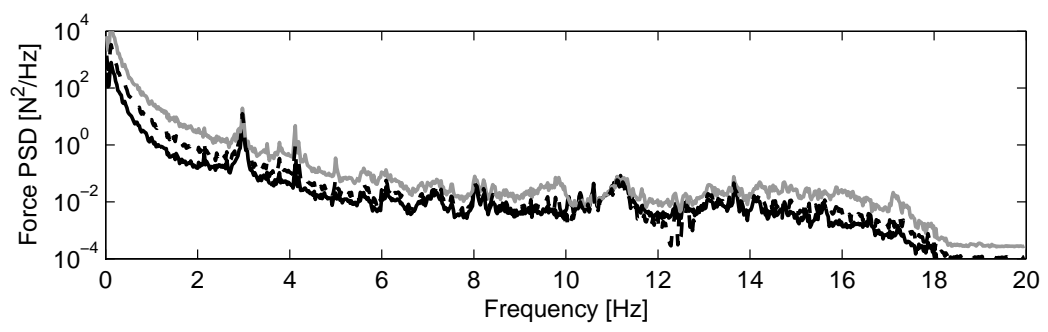

Fig. 10: Amplitude of the estimated PSD of the stochastic forces acting at node 39 (black solid line: auto-PSD vertical force, gray solid line: auto-PSD lateral force, black dashed line: cross-PSD vertical and lateral forces).

Due to ill-conditioning, it is not always possible to estimate the PSD of $n_{\mathrm{pS}}$ forces independently from Eq. (35). At a given frequency $\omega$, the number of structural modes significantly contributing to the response can become less than the number of forces to be determined. If this is the case, the rank of the FRF matrix $\mathbf{H}_{\mathrm{dp}_{\mathrm{S}}}(\omega)$ becomes less than $n_{\mathrm{p}}$ and small errors on the estimated PSD of the measured response $\mathbf{S}_{\mathrm{dd}}(\omega)$ may lead to large errors on the estimated force PSD. This is, for example, seen in Fig. 10, where the peak at a frequency of about $3 \mathrm{~Hz}$ is attributed to ill-conditioning near resonance. Other sources of uncertainty in the estimation of the force PSD include: errors on the estimated output PSD, the limited duration of the measurements, modeling errors, and discretization errors introduced by assuming a limited number of nodal forces. 
At this point, it is crucial to distinguish between (a) the noise statistics assumed for joint input-state estimation as characterized by the noise covariance matrices $\mathbf{Q}, \mathbf{R}$, and $\mathbf{S}$, and (b) the statistics of the actual noise processes, quantified by the PSD functions $\mathbf{S}_{\mathrm{p}_{\mathrm{S}} \mathrm{p}_{\mathrm{S}}}(\omega)$ and $\mathbf{S}_{\mathrm{v}_{\mathrm{M}} \mathrm{v}_{\mathrm{M}}}(\omega)$ (see Eq. (27)). Both these sets correspond to the same physical quantities - unknown stochastic excitation and measurement errors. The noise statistics $\mathbf{Q}, \mathbf{R}$, and $\mathbf{S}$ act as tuning parameters and determine the weighing of the data by the joint input-state estimation filtering algorithm (see also [34]). This weighing affects the uncertainty introduced by the (actual) noise processes. Although any choice is possible, the noise covariance matrices $\mathbf{Q}, \mathbf{R}$, and $\mathbf{S}$ are here estimated based on the following equation:

$$
\left[\begin{array}{cc}
\mathbf{Q} & \mathbf{S} \\
\mathbf{S}^{\mathrm{T}} & \mathbf{R}
\end{array}\right]=\left[\begin{array}{l}
\mathbf{B}^{\prime} \\
\mathbf{J}^{\prime}
\end{array}\right] \mathbf{C}_{\mathrm{p}}\left[\begin{array}{ll}
\mathbf{B}^{\prime \mathrm{T}} & \mathbf{J}^{\prime \mathrm{T}}
\end{array}\right]+\left[\begin{array}{cc}
\mathbf{0} & \mathbf{0} \\
\mathbf{0} & \mathbf{R}_{\mathrm{M}}
\end{array}\right]
$$

where $\mathbf{C}_{\mathrm{p}}$ is the covariance matrix of the identified stochastic forces $\mathbf{p}_{\mathrm{S}[k]}$ and is calculated as:

$$
\mathbf{C}_{\mathrm{p}}=\int_{\omega_{\min }}^{\omega_{\max }} \mathbf{S}_{\mathrm{p}_{\mathrm{S}} \mathrm{p}_{\mathrm{S}}}(\omega) \mathrm{d} \omega+\int_{-\omega_{\max }}^{-\omega_{\min }} \mathbf{S}_{\mathrm{p}_{\mathrm{S}} \mathrm{p}_{\mathrm{S}}}(\omega) \mathrm{d} \omega=2 \operatorname{Re}\left(\int_{\omega_{\min }}^{\omega_{\max }} \mathbf{S}_{\mathrm{p}_{\mathrm{S}} \mathrm{p}_{\mathrm{S}}}(\omega) \mathrm{d} \omega\right)
$$

The frequencies $\omega_{\min }$ and $\omega_{\max }$ define the frequency interval over which the covariance matrix $\mathbf{C}_{\mathrm{p}}$ is calculated. By using Eq. (36), the noise covariance matrices relate to the actual noise processes, i.e. ambient excitation and measurement noise. The boundaries of the frequency interval, $\omega_{\min }$ and $\omega_{\max }$, are chosen here as $\omega_{\min }=1 \times 2 \pi \mathrm{rad} / \mathrm{s}$ and $\omega_{\max }=16 \times 2 \pi \mathrm{rad} / \mathrm{s}$. The calculation of $\mathbf{C}_{\mathrm{p}}$ from Eq. (37) is performed by numerical integration.

For calculation of the measurement error covariance matrix $\mathbf{R}_{\mathrm{M}}$, a standard deviation of $7 \times$ $10^{-5} \mathrm{~m} / \mathrm{s}^{2}, 3 \times 10^{-5} \mathrm{~m} / \mathrm{s}^{2}$, and $6 \times 10^{-6} \mathrm{~m}$ is assumed for the error on the acceleration measurements obtained from the GMS-18 units, the uniaxial acceleration measurements, and the displacement measurements, respectively. The standard deviation for the error on the acceleration measurements is based on the resolution of the sensors as specified by the manufacturer. The standard deviation for the error on the displacement measurements exceeds the actual error standard deviation of the sensors (i.e. $\pm 1 \times 10^{-6} \mathrm{~m}$ ), thus accounting for inaccuracies due to horizontal motion of the tripod supports (see section 4.3). The measurement errors are assumed uncorrelated (i.e. $\mathbf{R}_{\mathrm{M}}$ is a diagonal matrix).

The force error PSD function $\mathbf{S}_{\tilde{p} \tilde{p}}(\omega)$ is now calculated from Eq. (27). The PSD function $\mathbf{S}_{\mathrm{P}_{\mathrm{S}} \mathrm{p}_{\mathrm{S}}}(\omega)$ used in the calculation was previously obtained from the experiment considering only ambient forces (Fig. 10). The PSD function of the measurement noise $\mathbf{S}_{\mathrm{v}_{M}} \mathrm{v}_{\mathrm{M}}(\omega)$ is obtained from Eq. (26), i.e. white measurement noise is assumed. As an alternative to calculating the force error PSD from Eq. (27), the actual force errors are calculated by applying the joint input-state estimation algorithm for the ambient vibration data considered before $\left(\mathbf{p}_{[k]}=0\right)$. Fig. 11 compares the PSD $\mathbf{S}_{\tilde{p} \tilde{p}}(\omega)$ of the error on the vertical forces at nodes 27 and 48 obtained using the two methods for calculating the error PSD; (1) from Eq. (27), and (2) by directly applying the joint input-state estimation algorithm for the ambient vibration data. The measured data is assumed to consist of the two accelerations and the two displacements introduced in Section 5 (i.e. a27ni, a48ni, d27z and d40z). The good agreement between the estimated error PSDs in Fig. 11 confirms the validity of the steady-state assumption that was made in the derivation of the underlying Eq. (27), as is also observed in [26].

The covariance of the force errors $\tilde{\mathbf{p}}_{[k \mid k]}$ is a measure for the overall uncertainty in the estimated 

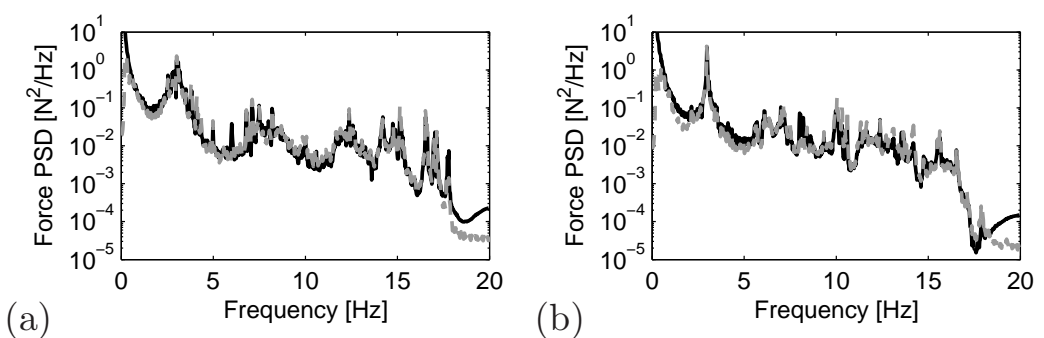

Fig. 11: PSD of the error on the identified vertical forces at (a) node 27 and (b) node 48, obtained using joint input-state estimation. The errors originate from unknown stochastic excitation, and measurement errors. The error obtained from the uncertainty quantification method is shown by a black solid line. The error obtained by applying the joint input-state estimation algorithm for a set of ambient measurement data is shown by a gray dashed line.

forces and is immediately obtained from the estimated force error PSD $\mathbf{S}_{\tilde{p} \tilde{p}}(\omega)$ :

$$
\operatorname{cov}\left(\tilde{\mathbf{p}}_{[k \mid k]}\right)=2 \operatorname{Re}\left(\int_{\omega_{\min }}^{\omega_{\max }} \mathbf{S}_{\tilde{\mathrm{p}} \tilde{\mathbf{p}}}(\omega) \mathrm{d} \omega\right)
$$

where $\omega_{\min }$ and $\omega_{\max }$ are the lower and upper bound, respectively, of a predefined frequency interval in which the uncertainty on the estimated forces has been quantified. These bounds are chosen as $\omega_{\min }=0.2 \times 2 \pi \mathrm{rad} / \mathrm{s}$ and $\omega_{\max }=16 \times 2 \pi \mathrm{rad} / \mathrm{s}$, and correspond to the cut-off frequencies of the filters applied in the data processing: a digital lowpass filter with a cut-off frequency of $16 \mathrm{~Hz}$ is applied to all measured signals before down-sampling (see also Section 3), and a digital highpass filter with a cut-off frequency of $0.2 \mathrm{~Hz}$ is applied to the estimated force signals in order to remove the low frequency force components that compensate for wind loads. Table 2 compares the estimated force error variance for two data sets: set 1 is the minimum set including two collocated accelerations and two displacements (4 sensors) that was introduced in Section 5; set 2 includes all response measurements on the bridge deck (40 sensors). The error variance for data set 2 is only slightly lower than the error variance for the minimum data set 1 . In this case, using an extensive data set only produces minor benefits.

\begin{tabular}{lll}
\hline & set 1 & set 2 \\
\hline Sensors & $\mathrm{d} 27 \mathrm{z}, \mathrm{d} 40 \mathrm{z}, \mathrm{a} 27 \mathrm{nni}, \mathrm{a} 48 \mathrm{ni}$ & $\mathrm{d} 27 \mathrm{z}, \mathrm{d} 40 \mathrm{z}, \mathrm{a} 27 \mathrm{ni}, \mathrm{a} 48 \mathrm{ni}, \mathrm{aj} \zeta$ \\
$\operatorname{Var}\left(\tilde{p}_{27[k \mid k]}\right)\left[\mathrm{N}^{2}\right]$ & 4.39 & 2.89 \\
$\operatorname{Var}\left(\tilde{p}_{48[k \mid k]}\right)\left[\mathrm{N}^{2}\right]$ & 6.86 & 6.58 \\
Total variance $\left[\mathrm{N}^{2}\right]$ & 11.25 & 9.47 \\
\hline
\end{tabular}

Table 2: Estimate of the steady-state force error variance (frequency range 0.2 to $16 \mathrm{~Hz}$ ) for two different sets of sensors. $\tilde{p}_{i[k \mid k]}$ represents the error on the time history of the vertical force at node $i$.

\section{Results force identification}

This section presents the results of the joint input-state estimation for the identification of impact, harmonic, and swept sine excitation applied to the bridge deck. The forces are estimated using the minimum data set consisting of two displacements and two accelerations introduced in Section 5. The noise covariance matrices $\mathbf{Q}, \mathbf{R}$, and $\mathbf{S}$ used in the force identification are identical to those computed in Section 6. The initial state estimate vector $\mathbf{x}_{[0 \mid-1]}$ and its error covariance matrix $\mathbf{P}_{[0 \mid-1]}$ are both assumed zero. 
Fig. 12 shows the results of the identification for a sequence of hammer impacts applied at nodes 27 and 48. A fairly good estimate of both forces is seen from both the time history and the frequency content. Three time intervals can be distinguished in Figs. 12b and 12e for a single hammer impact applied to the bridge deck; (1) the impact, (2) free vibration, and (3) ambient vibration. During the impact, the broadband hammer force excites the entire frequency range considered. The errors introduced by ambient forces (i.e. unknown stochastic forces) are small, since the hammer impact is far more important than the ambient loading. During the free vibration phase, the structure vibrates at its natural frequencies and modeling errors manifest in errors on the estimated force time history that generally decay exponentially over time. It is seen from Figs. 12a and 12d that this free vibration phase, characterized by force amplitudes that clearly decay exponentially over time, takes 30 to 40 seconds, depending on the amplitude of the hammer impact applied. After the free vibration phase, the measured response is predominantly due to ambient loads. The ambient vibration phase is, for example, seen in Figs. 12b and 12e for $t<104 \mathrm{~s}$. During this phase, the uncertainty on the estimated forces stems from ambient excitation and measurement errors. As expected, the force levels observed during this phase (i.e. the force error levels) are small and in line with the estimated error statistics obtained from the uncertainty quantification approach in Section 6 (see Table 2: $\sigma_{\tilde{p}_{27}}=\sqrt{4.39 \mathrm{~N}^{2}}=2.10 \mathrm{~N}, \sigma_{\tilde{p}_{48}}=\sqrt{6.86 \mathrm{~N}^{2}}=2.62 \mathrm{~N}$ ). It is concluded that the errors introduced by the ambient excitation and the measurement errors are small compared to the peak values generated by the impact forces. From the time history of the forces in Figs. 12b and $12 \mathrm{e}$, it is also seen that, in this case of broadband excitation, the algorithm is able to properly distinguish between the two forces. By comparing the frequency spectrum of the measured and estimated forces, e.g. in Fig. 12c, it is seen that the error in the estimated force is generally small in those frequency ranges where the forward simulations are in good agreement with the measured response of the footbridge (see also Fig. 7).

Fig. 13 shows the results for the identification procedure for two harmonic forces, applied at nodes 27 and 48. The dominant excitation frequency of the force applied at node 27 is $8 \mathrm{~Hz}$, whereas the dominant excitation frequency of the force applied at node 48 is $6 \mathrm{~Hz}$. In this case where the excitation is dominated by a limited number of frequencies, modeling errors occurring at the excitation frequencies prohibit a proper distinction between the two independent forces. The identified force at node 48 clearly contains an important harmonic component at $8 \mathrm{~Hz}$, which is less pronounced in the measured force signal.

Fig. 14 shows the results of the identification procedure for two swept sine forces, applied at nodes 27 and 48. The excitation frequency of the force applied at node 27 rises from $0.5 \mathrm{~Hz}$ to $10 \mathrm{~Hz}$ in $285 \mathrm{~s}$, whereas for node 48, the excitation frequency rises from $0.375 \mathrm{~Hz}$ to $7.5 \mathrm{~Hz}$ in the same time period. The excitation frequency of the forces applied at nodes 27 and 48 is increased in steps of $1 \mathrm{mHz}$ and $0.75 \mathrm{mHz}$ every $30 \mathrm{~ms}$, respectively. Two cycles of $285 \mathrm{~s}$ are considered. The response of the structure depends on the rate at which the frequency increases, i.e. $1 / 30 \mathrm{~Hz} / \mathrm{s}$ for the force applied at node 27 and $1 / 40 \mathrm{~Hz} / \mathrm{s}$ for the force applied at node 48 . For each frequency step, the response of the structure evolves from harmonic vibration at the previous excitation frequencies to harmonic vibration at the current excitation frequencies. When the excitation frequency is slowly increased, the response achieves steady-state before the excitation frequency is changed again, whereas if the excitation frequency is rapidly increased, the response is mainly dominated by transient phenomena. In the transition phase, the structure primarily vibrates at its natural frequencies and the errors are comparable to those observed in the free vibration phase following impact excitation. In addition, modeling errors at the excitation frequency result in errors on 
the estimated stationary forces. As in the case of harmonic excitation, modeling errors prohibit a proper distinction between the two forces, resulting in large errors on the estimated forces for some frequencies.
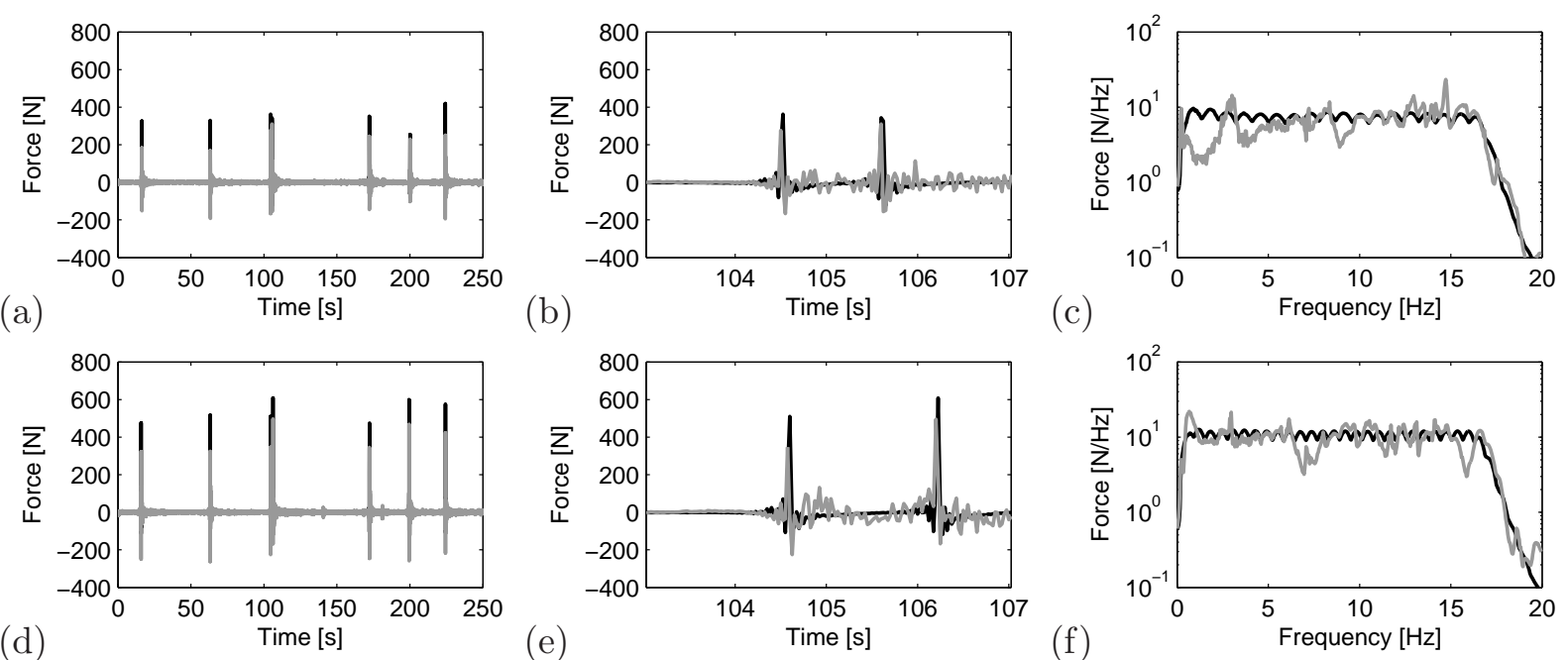

Fig. 12: Time history (left), detail of the time history (middle), and averaged amplitude of the narrow band frequency spectrum (right) of the impact forces applied at node $27((\mathrm{a})-(\mathrm{c}))$ and node $48((\mathrm{~d})-(\mathrm{f}))$, sensor set 1 . The measured force signals are shown in black, the identified force signals are shown in gray.
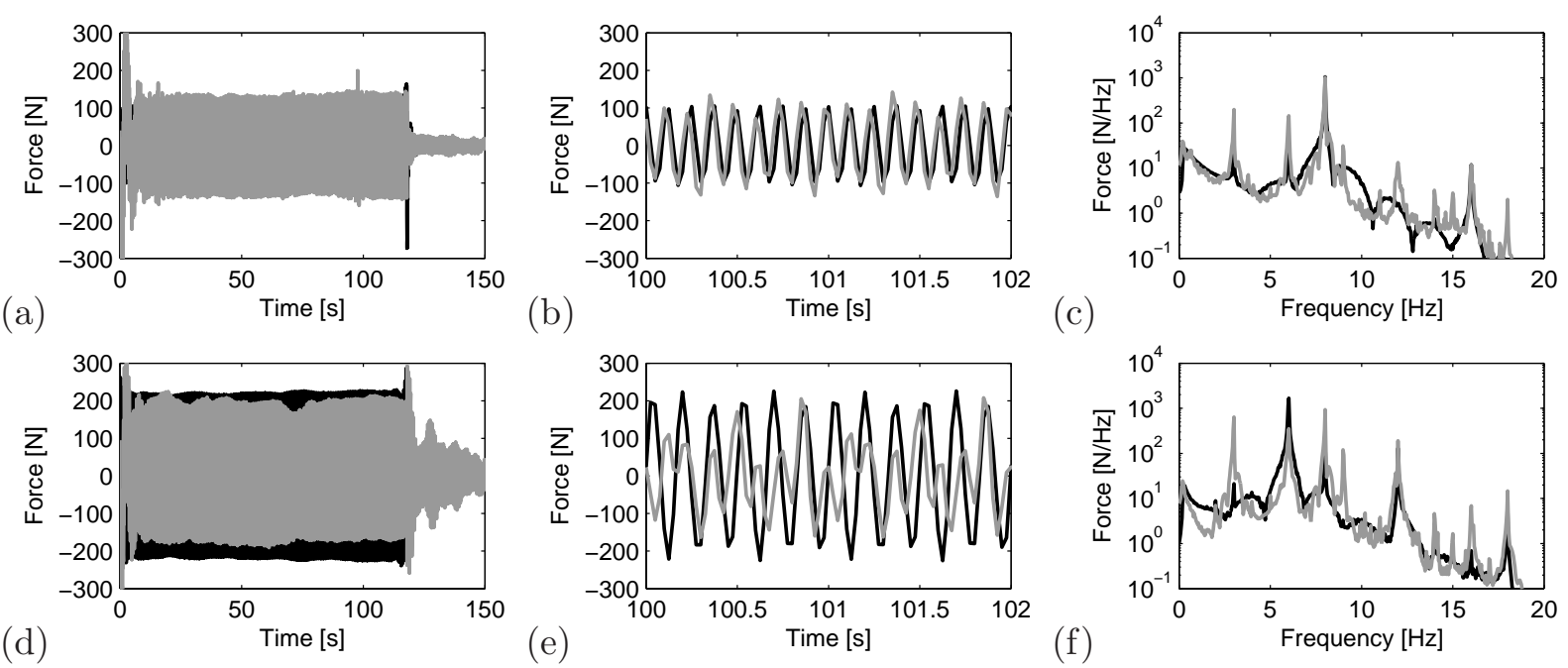

Fig. 13: Time history (left), detail of the time history (middle), and averaged amplitude of the narrow band frequency spectrum (right) of the harmonic forces applied at node $27((\mathrm{a})-(\mathrm{c}))$ and node $48((\mathrm{~d})-(\mathrm{f}))$, sensor set 1 . The measured force signals are shown in black, the identified force signals are shown in gray. 

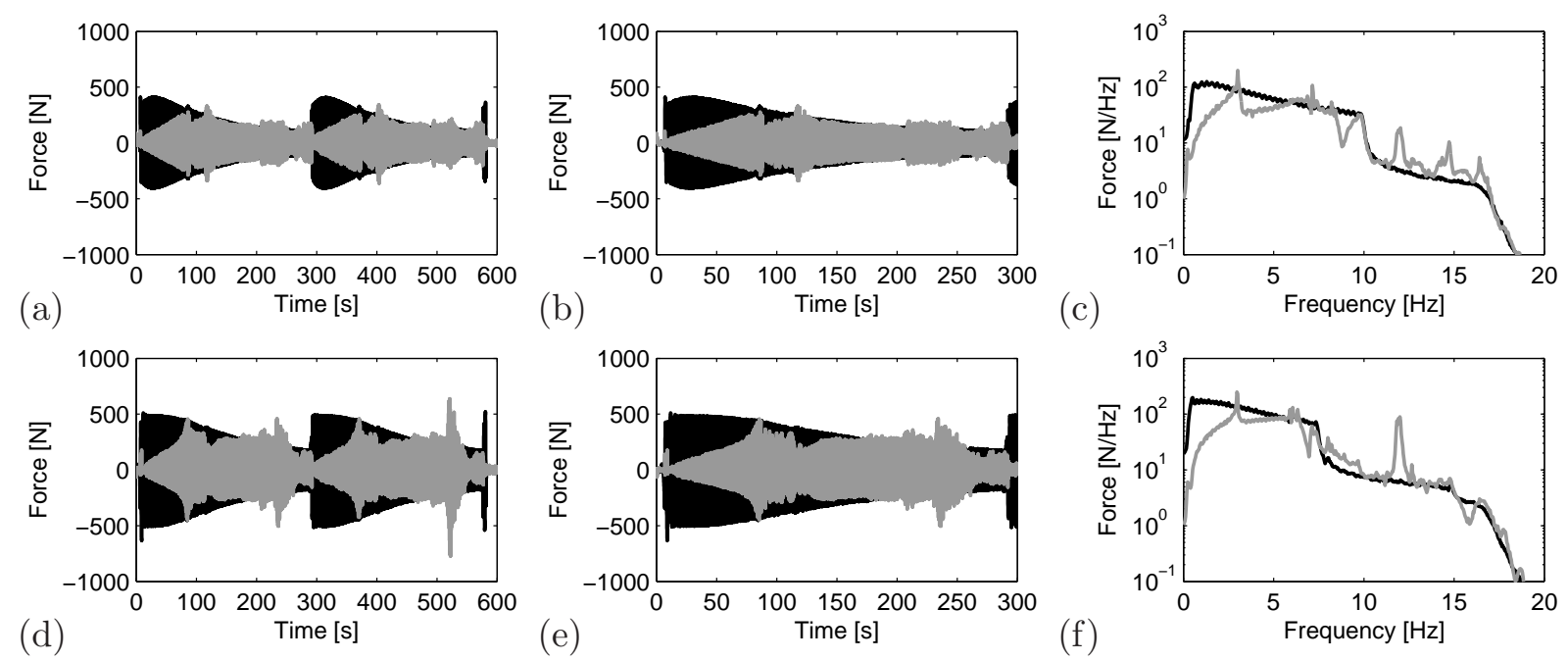

Fig. 14: Time history (left), detail of the time history (middle), and averaged amplitude of the narrow band frequency spectrum (right) of the swept sine forces applied at node $27((\mathrm{a})-(\mathrm{c}))$ and node $48((\mathrm{~d})-(\mathrm{f}))$, sensor set 1 . The measured force signals are shown in black, the identified force signals are shown in gray.

\section{Conclusions}

This paper presents a verification of a joint input-state estimation algorithm, using data obtained from in situ experiments on a footbridge. The joint input-state estimation algorithm is used for the identification of impact, harmonic, and swept sine forces applied to the bridge deck. A dynamic model of the structure has been composed using a detailed finite element model of the structure, which was updated using a set of experimental modal characteristics. Numerical simulations show good overall agreement with the true dynamic behavior of the footbridge. The noise processes considered in the joint input-state estimation mainly cover unknown stochastic excitation acting on the structure, in addition to the forces to be identified. The unknown stochastic excitation in the present case primarily consists of wind loads that give rise to uncertainty in the estimated forces. The uncertainty is quantified based on the power spectral density of the ambient forces, which is identified from the response of the structure under ambient loading. Verification of the results is carried out by comparing the estimated forces with the actual measured forces. For the case of broadband impact loading, the forces obtained from joint input-state estimation are in good agreement with the true, measured forces. Although good overall agreement is also observed between the estimated and measured forces for harmonic and swept sine loads, modeling errors in this case are found to prohibit a proper distinction between the multiple independent forces.

\section{Acknowledgments}

The research presented in this paper has been performed within the framework of the project G.0738.11 "Inverse identification of wind loads on structures", funded by the Research Foundation Flanders (FWO), Belgium. Their financial support is gratefully acknowledged. The authors affiliated to KU Leuven are all members of the KU Leuven - BOF PFV/10/002 OPTEC - Optimization in Engineering Center. 


\section{References}

[1] P. Guillaume, E. Parloo, G. De Sitter, Source identification from noisy response measurements using an iterative weighted pseudo-inverse approach., in: Proceedings of ISMA2002 International Conference on Noise and Vibration Engineering, Leuven, Belgium, pp. 1817-1824.

[2] Y. Liu, W. Shepard, Dynamic force identification based on enhanced least squares and total least squares schemes in the frequency domain, Journal of Sound and Vibration 282 (2005) 37-60.

[3] E. Parloo, P. Guillaume, M. Van Overmeire, Damage assessment using mode shape sensitivities, Mechanical Systems and Signal Processing 17 (2003) 499-518.

[4] M. Klinkov, C. Fritzen, An updated comparison of the force reconstruction methods, Key Engineering Materials 347 (2007) 461-466.

[5] L. Nordström, T. Nordberg, A critical comparison of time domain load identification methods, in: Proceedings of the 6th International Conference on Motion and Vibration Control, Saitama, Japan, pp. 1151-1156.

[6] E. Jacquelin, A. Bennani, P. Hamelin, Force reconstruction: analysis and regularization of a deconvolution problem, Journal of Sound and Vibration 265 (2003) 81-107.

[7] D. Bernal, A. Ussia, Sequential deconvolution input reconstruction, Mechanical Systems and Signal Processing 50-51 (2015) 41-55.

[8] J. Hadamard, Lectures on Cauchy's Problem in Linear Partial Differential Equations., Yale University Press, New Haven, CT, 1923.

[9] E. Hernandez, D. Bernal, State estimation in structural systems with model uncertainties, ASCE Journal of Engineering Mechanics 134 (2008) 252-257.

[10] E. Hernandez, A natural observer for optimal state estimation in second order linear structural systems, Mechanical Systems and Signal Processing 25 (2011) 2938-2947.

[11] J. Ching, J. Beck, Real-time reliability estimation for serviceability limit states in structures with uncertain dynamic excitation and incomplete output data, Probabilistic Engineering Mechanics 22 (2007) 50-62.

[12] C. Papadimitriou, C.-P. Fritzen, P. Kraemer, E. Ntotsios, Fatigue predictions in entire body of metallic structures from a limited number of vibration sensors using Kalman filtering, Structural Control and Health Monitoring 18 (2011) 554-573. Published online in Wiley InterScience (www.interscience.wiley.com). DOI:10.1002/stc.395.

[13] M. Wu, A. Smyth, Application of the unscented Kalman filter for real-time nonlinear structural system identification, Structural Control and Health Monitoring 14 (2007) 971-990.

[14] E. Chatzi, A. Smyth, The unscented Kalman filter and particle filter methods for nonlinear structural system identification with non-collocated heterogeneous sensing, Structural Control and Health Monitoring 16 (2009) 99-123.

[15] S. Mariani, A. Corigliano, Impact induced composite delamination: state and parameter identification via joint and dual extended kalman filters, Computer Methods in Applied Mechanics and Engineering 194 (2005) $5242-5272$.

[16] J. Ching, J. Beck, K. Porter, Bayesian state and parameter estimation of uncertain dynamical systems, Probabilistic Engineering Mechanics 21 (2006) 81-96.

[17] E. Chatzi, A. Smyth, Nonlinear system identification: Particle-based methods, in: M. Beer, I. Kougioumtzoglou, E. Patelli, I.-K. Au (Eds.), Encyclopedia of Earthquake Engineering, Springer, London, UK, 2014.

[18] M. Klinkov, C.-P. Fritzen, Online estimation of external loads from dynamic measurements, in: P. Sas, M. D. Munck (Eds.), Proceedings ISMA2006, Leuven, Belgium, pp. 3957-3968.

[19] C. Hsieh, Robust two-stage Kalman filters for systems with unknown inputs, IEEE Transactions on Automatic Control 45 (2000) 2374-2378.

[20] C.-S. Hsieh, Extension of unbiased minimum-variance input and state estimation for systems with unknown inputs, Automatica 45 (2009) 2149-2153.

[21] C.-S. Hsieh, On the global optimality of unbiased minimum-variance state estimation for systems with unknown inputs., Automatica 46 (2010) 708-715.

[22] C.-S. Hsieh, Optimal filtering for systems with unknown inputs via the descriptor Kalman filtering method., Automatica 47 (2011) 2313-2318.

[23] S. Eftekhar Azam, C. Papadimitriou, E. Chatzi, A dual Kalman filter approach for state estimation via outputonly acceleration measurements, Mechanical Systems and Signal Processing 60-61 (2015) 866-886.

[24] S. Gillijns, B. De Moor, Unbiased minimum-variance input and state estimation for linear discrete-time systems with direct feedthrough, Automatica 43 (2007) 934-937.

[25] E. Lourens, C. Papadimitriou, S. Gillijns, E. Reynders, G. De Roeck, G. Lombaert, Joint input-response 
estimation for structural systems based on reduced-order models and vibration data from a limited number of sensors, Mechanical Systems and Signal Processing 29 (2012) 310-327.

[26] K. Maes, A. Smyth, G. De Roeck, G. Lombaert, Joint input-state estimation in structural dynamics, Mechanical Systems and Signal Processing 70-71 (2016) 445-466.

[27] Y. Niu, M. Klinkov, C.-P. Fritzen, Online force reconstruction using an unknown-input Kalman filter approach., in: Proceedings of the 8th International Conference on Structural Dynamics, EURODYN 2011, Leuven, Belgium, pp. 2569-2576.

[28] E. Lourens, E. Reynders, G. De Roeck, G. Degrande, G. Lombaert, An augmented Kalman filter for force identification in structural dynamics, Mechanical Systems and Signal Processing 27 (2012) 446-460.

[29] K. Maes, E. Lourens, K. Van Nimmen, E. Reynders, G. De Roeck, G. Lombaert, Design of sensor networks for instantaneous inversion of modally reduced order models in structural dynamics, Mechanical Systems and Signal Processing 52-53 (2015) 628-644.

[30] E. Chatzi, C. Fuggini, Structural identification of a super-tall tower by GPS and accelerometer data fusion using a multi-rate Kalman filter, in: F. Strauss, Bergmeister (Eds.), Proceedings of IALCCE 2014,4th International Symposium on Life-Cycle Civil Engineering, Tokyo, Japan, pp. 144-151.

[31] F. Naets, J. Cuadrado, W. Desmet, Stable force identification in structural dynamics using kalman filtering and dummy-measurements, Mechanical Systems and Signal Processing 50-51 (2015) 235-248.

[32] P. Van Overschee, B. De Moor, Subspace algorithm for the stochastic identification problem, Automatica 29 (1993) 649-660.

[33] E. Reynders, G. De Roeck, Reference-based combined deterministic-stochastic subspace identification for experimental and operational modal analysis, Mechanical Systems and Signal Processing 22 (2008) 617-637.

[34] K. Maes, A. Smyth, G. De Roeck, G. Lombaert, Uncertainty quantification for joint input-state estimation in structural dynamics, in: M. Papadrakakis, V. Papadopoulos, V. Plevris (Eds.), Proceedings of the 1st International Conference on Uncertainty Quantification in Computational Sciences and Engineering, UNCECOMP 2015, Crete Island, Greece.

[35] K. Van Nimmen, G. Lombaert, G. De Roeck, P. Van den Broeck, Vibration serviceability of footbridges: Evaluation of the current codes of practice, Engineering Structures 59 (2014) 448-461.

[36] K. Deckers, P. Guillaume, D. Lefeber, G. De Roeck, E. Reynders, Modal testing of bridges using low-weight pneumatic artificial muscle actuators, in: Proceedings of IMAC 26, the International Modal Analysis Conference, Orlando, FL. CD-ROM.

[37] Basic Analysis Guide, ANSYS Release 11.0, ANSYS Inc., 2007.

[38] B. Peeters, G. De Roeck, Reference-based stochastic subspace identification for output-only modal analysis, Mechanical Systems and Signal Processing 13 (1999) 855-878.

[39] MATLAB Optimization Toolbox User's Guide, The MathWorks, 2011.

[40] E. Reynders, System identification methods for (operational) modal analysis: review and comparison, Archives of Computational Methods in Engineering 19 (2012) 51-124.

[41] ENV1991-2-4:1995 Eurocode 1: Basis of design and actions on structures - Part 2-4: Actions on structures Wind actions, European Committee for Standardization, 1995. 